\title{
Ultradifferentiable CR Manifolds
}

\author{
Stefan Fürdös ${ }^{1,2}$ (1)
}

Received: 3 August 2018 / Published online: 18 April 2019

(c) The Author(s) 2019

\begin{abstract}
In this article, the notion of ultradifferentiable CR manifold is introduced and an ultradifferentiable regularity result for finitely nondegenerate CR mappings is proven. Here, ultradifferentiable means with respect to Denjoy-Carleman classes defined by weight sequences. Furthermore, the regularity of infinitesimal CR automorphisms on ultradifferentiable abstract CR manifolds is investigated.
\end{abstract}

Keywords Ultradifferentiable CR manifolds · Ultradifferentiable regularity · CR mappings $\cdot$ Infinitesimal CR automorphisms

Mathematics Subject Classification $32 \mathrm{~V} 05 \cdot 32 \mathrm{~V} 99 \cdot 26 \mathrm{E} 10 \cdot 35 \mathrm{~A} 18$

\section{Introduction}

The primary focus of this article is the study of the regularity of CR mappings. Looking at the literature concerning this problem, one observes that most theorems about the regularity of CR mappings are of a similar form which can be summarised as follows: We consider a CR mapping $H$ between two CR submanifolds $M$ and $M^{\prime}$ with some a priori regularity that extends to a holomorphic mapping defined on a wedge with edge $M$. If the mapping and/or the manifolds satisfy certain nondegeneracy conditions at some point then it is proven that $H$ is actually of optimal regularity near this point, that is smooth if $M$ and $M^{\prime}$ are smooth, or real analytic if the manifolds are real analytic. We should mention that the nondegeneracy assumptions are heavily tailored towards the methods applied in the various different proofs. In particular, it is worth noting that in most instances the conditions in the smooth setting differ sharply from those used in the analytic category. In the case of smooth CR manifolds, the fundamental contributions

Stefan Fürdös

stefan.fuerdoes@univie.ac.at

1 Faculty of Mathematics, University of Vienna, Oskar-Morgenstern-Platz 1, 1090 Vienna, Austria

2 Present Address: Department of Mathematics and Statistics, Masaryk University, Kotlarska 2, 61137 Brno, Czech Republic 
are the pioneering works of Fefferman [13] and Nirenberg-Webster-Yang [28]. We should also notice that in the analytic setting surprisingly weak assumptions often suffice, c.f. e.g. the classical results of Baouendi-Jacobowitz-Treves [28], Huang [20] and Pinčuk [30].

One of the rare cases, where under the identical assumptions it has been possible to show that $H$ is smooth if the manifolds are smooth and analytic if $M$ and $M^{\prime}$ are both analytic manifolds, has been the results of Lamel [23,24]. He proved that every finitely nondegenerate CR mapping between two generic submanifolds that extends holomorphically is smooth and even analytic if both manifolds are real analytic.

Recently, Berhanu-Xiao [3] were able to strengthen this result in the smooth case by relaxing partially its assumptions. They require only the target manifold to be an embedded CR manifold, the source manifold could be only an abstract CR manifold. The finitely nondegenerate condition on the mapping remains unchanged but the holomorphic extension obviously makes no sense in this situation. It is replaced in the theorem of Berhanu-Xiao with the assumption that the fibres of the wavefront set of $H$ do not include opposite directions.

This microlocal assumption is automatically satisfied in the embedded setting if extension to a wedge is assumed since Baouendi-Chang-Treves [1] showed that for $\mathrm{CR}$ distributions on $\mathrm{CR}$ submanifolds of $\mathbb{C}^{N}$, the holomorphic extension into wedges is in fact a microlocal condition, which they used to define the hypoanalytic wavefront set of CR distributions. It coincides with the analytic wavefront set if the manifold is analytic. If the manifold is only smooth then the hypoanalytic wavefront set includes the smooth wavefront set.

Since the results of Lamel and Berhanu-Xiao suggest that finite nondegeneracy preserves regularity quite well, the following question arises naturally. Given a subsheaf $\mathcal{A}$ of the sheaf of smooth functions, we may ask that if in the formulation of the theorem of Lamel the manifolds are assumed to be of class $\mathcal{A}$, does it follow that the $\mathrm{CR}$ mapping has to be of class $\mathcal{A}$ as well?

Of course, we have to assume that $\mathcal{A}$ satisfies certain properties. First of all, in order for the conjecture above to make sense, $\mathcal{A}$ must be closed under composition and the implicit function theorem must hold in the category of mappings of class $\mathcal{A}$. Furthermore, if we try to modify the existing proofs in the smooth category then we need some version of $\mathcal{A}$-wavefront set or more precisely a definition of $\mathcal{A}$-microlocal regularity. We should note at this point that in both Lamel's proof and that of BerhanuXiao the characterisation of the smooth wavefront set by almost-analytic extensions was heavily used as both relied on an almost-analytic version of the implicit function theorem.

We are mainly interested in subsheafs of smooth functions that contain strictly the sheaf of real-analytic functions. We shall call the elements of such sheafs ultradifferentiable functions. Generally ultradifferentiable functions are determined either by estimates on its derivatives or its Fourier transform. The most well-known examples of ultradifferentiable classes are the Gevrey classes, see e.g. [32].

Here, we consider the category of the so-called Denjoy-Carleman classes, which are defined in the following way. If $\mathcal{M}=\left(m_{j}\right)_{j}$ is a sequence of positive real numbers then the Denjoy-Carleman class associated with $\mathcal{M}$ consists of those smooth functions that satisfy the following generalised Cauchy estimate 


$$
\left|\partial^{\alpha} f(x)\right| \leq C h^{|\alpha|} m_{|\alpha|}|\alpha| !
$$

on compact sets, where $C$ and $h$ are constants independent of $\alpha$. We will also say that a smooth function $f$ obeying (1.1) is of class $\{\mathcal{M}\}$. In particular, if $\mathcal{M}=\left(j !^{s}\right)_{j}$ then the associated Denjoy-Carleman class to $\mathcal{M}$ is the Gevrey class of order $s+1$.

Examining literature concerning the Denjoy-Carleman classes and their properties, one can observe that stability conditions of the associated class correlate with properties of the weight sequence. For example, we know that, if $\mathcal{M}$ is a regular weight sequence in the sense of [11], then the Denjoy-Carleman class associated with $\mathcal{M}$ is closed under composition, solving ordinary differential equations and the implicit function theorem holds in the class, c.f. e.g. [31]. Hence for regular sequences $\mathcal{M}$, we can consider manifolds of Denjoy-Carleman type. We shall say such a manifold is an ultradifferentiable manifold of class $\{\mathcal{M}\}$.

On the other hand, Hörmander [18] introduced the ultradifferentiable wavefront set for distributions defined on open subsets of the Euclidean space. But since he worked under comparatively weak conditions on the weight sequence, Hörmander was only able to define the ultradifferentiable wavefront set $\mathrm{WF}_{\mathcal{M}} u$ of distributions $u$ on realanalytic manifolds but not distributions defined on ultradifferentiable manifolds.

However, using Dyn'kins characterisation of ultradifferentiable functions by almost-analytic extensions [10,11], we were able in [14] to develop a geometric theory for the ultradifferentiable wavefront set. In particular, if the weight sequence is regular, the ultradifferentiable wavefront set of a distribution on an ultradifferentiable manifold is shown to be well defined. If we put additional conditions on the weight sequence, then we have also shown in [14] a microlocal elliptic regularity result for linear partial differential operators with ultradifferentiable coefficients acting on ultradifferentiable vector bundles. We are going to call weight sequences, that satisfy these conditions, normal.

With these results at hand and an $\mathcal{M}$-almost-analytic version of the almost-analytic implicit function theorem used in Lamel [24] and Berhanu-Xiao [3], it is possible to prove the ultradifferentiable version of the regularity result of Lamel:

Theorem 1.1 Let $\mathcal{M}$ be a normal weight sequence and $M \subseteq \mathbb{C}^{N}, M^{\prime} \subseteq \mathbb{C}^{N^{\prime}}$ be two generic ultradifferentiable submanifolds of class $\{\mathcal{M}\}, p_{0} \in M, p_{0}^{\prime} \in M^{\prime}$ and $H:\left(M, p_{0}\right) \rightarrow\left(M^{\prime}, p_{0}^{\prime}\right)$ a $\mathcal{C}^{k_{0}}-C R$ mapping that is $k_{0}$-nondegenerate at $p_{0}$. Suppose furthermore that $H$ extends continuously to a holomorphic map in a wedge $\mathcal{W}$ with edge $M$. Then $H$ is ultradifferentiable of class $\{\mathcal{M}\}$ in a neighbourhood of $p_{0}$.

For the definition of finite nondegeneracy of a CR mapping, we refer to the beginning of Sect. 5 and for the definition of normal weight sequence to Sect. 2.

More precisely this paper is structured as follows. In Sect. 2, the necessary results on Denjoy-Carleman classes and ultradifferentiable manifolds are discussed. In Sect. 3, we first recall the results from Dyn'kin $[10,11]$ on the almost-analytic extension of ultradifferentiable functions. Furthermore, we give the definition of the ultradifferentiable wavefront set according to Hörmander [19] and close the section by briefly discussing the results on the ultradifferentiable wavefront set from [14], that are needed later on. 
In Sect. 4, basic definitions and first results on ultradifferentiable CR manifolds are given, whereas the proofs of Theorem 1.1 and of ultradifferentiable versions of other regularity results of Lamel and Berhanu-Xiao are presented in Sect. 5. The last section is devoted to present essentially the generalisation of [15] concerning the smoothness of infinitesimal CR automorphisms to normal Denjoy-Carleman classes. We end by examining smooth infinitesimal CR automorphisms on formally holomorphic nondegenerate quasianalytic CR submanifolds.

We should note that although Theorem 1.1 gives rather precise information on the regularity of the mapping under consideration, the assumption of normality on the weight sequence is an obstruction, in the sense that any Denjoy-Carleman class given by a normal weight sequence is contained in some Gevrey class, c.f. [34]. It is possible to overcome this obstacle, but for that we have to work in a far more general setting, which will be done in a forthcoming paper.

\section{Denjoy-Carleman Classes}

In this section, we summarise the results for Denjoy-Carleman classes that we need throughout the paper. For a more detailed presentation, see [14]. Note that, unless stated otherwise, $\Omega \subseteq \mathbb{R}^{n}$ will be an open set.

Definition 2.1 Let $\mathcal{M}=\left(m_{k}\right)_{k}$ be a sequence of positive numbers. Then $\mathcal{M}$ is a regular weight sequence iff the following conditions hold.

$$
\begin{gathered}
m_{0}=m_{1}=1 \\
\sup _{k} \sqrt[k]{\frac{m_{k+1}}{m_{k}}}<\infty \\
m_{k}^{2} \leq m_{k-1} m_{k+1} \quad k \in \mathbb{N} \\
\lim _{k \rightarrow \infty} \sqrt[k]{m_{k}}=\infty
\end{gathered}
$$

If $\mathcal{M}$ satisfies (M1), (M3), (M4) and

$$
m_{j+k} \leq C q^{j+k} m_{j} m_{k} \quad \forall(j, k) \in \mathbb{N}_{0}^{2}
$$

for some constants $C, q>0$, then we say that $\mathcal{M}$ is a normal weight sequence.

Note that a normal weight sequence is regular, since (M2') implies (M2), c.f. [21]. We shall also mention, that we assume that the weight sequence is normal only in those statements, whose proofs actually require the stronger condition on the weight sequence. 
Definition 2.2 Let $\mathcal{M}$ be a regular weight sequence. Then we say that a smooth function $f \in \mathcal{E}(\Omega)$ is ultradifferentiable of class $\{\mathcal{M}\}$ iff for all compact sets $K \subseteq \Omega$ there are constants $C, h>0$ such that

$$
\left|\partial^{\alpha} f(x)\right| \leq C h^{|\alpha|} m_{|\alpha|}|\alpha| !
$$

for all $x \in K$. The space of all ultradifferentiable functions of class $\{\mathcal{M}\}$ is denoted by $\mathcal{E}_{\mathcal{M}}(\Omega)$. It is sometimes also called the Denjoy-Carleman class associated with $\mathcal{M}$.

We say also that a mapping $F=\left(F_{1}, \cdots, F_{m}\right): \Omega \rightarrow \mathbb{R}^{m}$ is of class $\{\mathcal{M}\}$, if all components $F_{j}$ are of class $\{\mathcal{M}\}$.

Example 2.3 If $s>0$ consider the normal weight sequence $\mathcal{M}^{s}=\left(k !^{s}\right)_{k}$. Its associated Denjoy-Carleman class is the Gevrey class $\mathcal{G}^{s+1}(\Omega)=\mathcal{E}_{\mathcal{M}^{s}}(\Omega)$ of order $s+1$ on $\Omega$, c.f. [32].

On the other hand, the constant sequence $\mathcal{M}^{0}=(1)_{k}$ gives the space $\mathcal{O}(\Omega)$ of real-analytic functions on $\Omega$. Note that $\mathcal{M}^{0}$ is neither normal nor regular in the sense of Definition 2.1.

If $\mathcal{M}$ and $\mathcal{N}=\left(n_{k}\right)_{k}$ are two regular weight sequences then we write $\mathcal{M} \preccurlyeq \mathcal{N}$ iff there is a constant $Q$ such that $m_{k} \leq Q^{k+1} n_{k}$. It holds that $\mathcal{E}_{\mathcal{M}} \subseteq \mathcal{E}_{\mathcal{N}}$ if and only if $\mathcal{M} \preccurlyeq \mathcal{N}$. Thus we see that (M4) means that $\mathcal{O} \subsetneq \mathcal{E}_{\mathcal{M}}$ and (M2) implies that $\mathcal{E}_{\mathcal{M}}$ is closed under derivation, i.e. if $f \in \mathcal{E}_{\mathcal{M}}(\Omega)$ then $\partial^{\alpha} f \in \mathcal{E}_{\mathcal{M}}(\Omega)$ for all multi-indices $\alpha \in \mathbb{N}_{0}^{n}$. Furthermore, we have

Lemma 2.4 (c.f. Remark 2.5 in [14]) Let the Denjoy-Carleman class $\mathcal{E}_{\mathcal{M}}$ be closed under derivation closed and suppose that $f \in \mathcal{E}_{\mathcal{M}}(\Omega)$ and $f\left(x_{1}, \ldots, x_{j-1}, a, x_{j+1}\right.$, $\left.\ldots, x_{n}\right)=0$ for some fixed $a \in \mathbb{R}$ and all $x_{k}, k \neq j$, with the property that $\left(x_{1}, \ldots, x_{j-1}, a, x_{j+1}, \ldots, x_{n}\right) \in \Omega$. Then there exists some $g \in \mathcal{E}_{\mathcal{M}}(\Omega)$ such that

$$
f(x)=\left(x_{j}-a\right) g(x) .
$$

In fact, if $\mathcal{M}$ is a regular weight sequence then the associated Denjoy-Carleman class satisfies a number of further stability properties, see e.g. [4]. In particular, if $f \in \mathcal{E}_{\mathcal{M}}$ and $G$ is a mapping of class $\{\mathcal{M}\}$ then $1 / f \in \mathcal{E}_{\mathcal{M}}$ and $f \circ G \in \mathcal{E}_{\mathcal{M}}$ whenever defined. Furthermore, the inverse function and implicit function theorems hold in the category of regular Denjoy-Carleman classes.

Therefore, we can define manifolds, vector bundles, vector fields, differential forms of class $\{\mathcal{M}\}$ as in the smooth (or analytic) category.

Remark 2.5 Let $E$ be an ultradifferentiable vector bundle of class $\{\mathcal{M}\}$. Then $E$ can also be considered as a smooth vector bundle or as a vector bundle of class $\mathcal{N}$ for any weight sequence $\mathcal{N} \succcurlyeq \mathcal{M}$. We observe in particular that a local basis of $\mathcal{E}_{\mathcal{M}}(M, E)$ is also a local basis of $\mathcal{E}_{\mathcal{N}}(M, E)$ and $\mathcal{E}(M, E)$, respectively.

We denote by $\mathfrak{X}_{\mathcal{M}}(M)=\mathcal{E}_{\mathcal{M}}(M, T M)$ the Lie algebra of ultradifferentiable vector fields on $M$. Note that $\mathcal{E}_{\mathcal{M}}$ is closed under solving ODEs if $\mathcal{M}$ is a regular weight 
sequence, see [22] and [38], and therefore an integral curve of an ultradifferentiable vector field of class $\{\mathcal{M}\}$ is an $\mathcal{E}_{\mathcal{M}}$-curve.

The next result is the ultradifferentiable version of Sussmann's Theorem [33].

Theorem 2.6 Let $p_{0} \in \Omega$ and a collection $\mathfrak{D}$ of ultradifferentiable vector fields of class $\{\mathcal{M}\}$. Then there exists an ultradifferentiable submanifold $W$ of $\Omega$ through $p_{0}$ such that all vector fields in $\mathfrak{D}$ are tangent to $W$ at all points of $W$ and such that the following holds:

(1) The germ of $W$ at $p_{0}$ is unique, i.e. if $W^{\prime}$ is an ultradifferentiable submanifold of $\Omega$ containing $p_{0}$ and to which all vector fields of $\mathfrak{D}$ are tangent at every point of $W^{\prime}$ then there is a neighbourhood $V \subseteq \Omega$ of $p_{0}$ such that $W \cap V \subseteq W^{\prime} \cap V$.

(2) For every open set $U \subseteq \Omega$ containing $p_{0}$, there exists $J \in \mathbb{N}$ and open neighbourhoods $V_{1} \subseteq V_{2} \subseteq U$ of $p_{0}$ such that every point $p \in W \cap V_{1}$ can be reached from $p_{0}$ by a polygonal path of $J$ integral curves of vector fields in $\mathfrak{D}$ contained in $W \cap V_{2}$.

The proof of Theorem 2.6 is essentially the same as in the smooth setting, c.f. e.g. [2], due to the fact that the solution of a (Banach space valued) ODE with ultradifferentiable data depends ultradifferentiable on said data, c.f. [38]. The (unique) germ of the manifold $W$ will be denoted as the local Sussmann orbit of $p_{0}$ relative to $\mathfrak{D}$. The local Sussmann orbit does not depend on $\Omega$.

One of the main differences between the space of smooth functions and the space of real-analytic functions is that in the smooth case there exist nontrivial test functions $\varphi \in \mathcal{D}(\Omega)$, whereas $\mathcal{D} \cap \mathcal{O}=\{0\}$. Since the existence of nontrivial test functions is equivalent to the existence of nonzero flat functions, it makes sense to give the following definition in the ultradifferentiable setting.

Definition 2.7 Let $E \subseteq \mathcal{E}(\Omega)$ be a subalgebra. We say that $E$ is quasianalytic iff for $f \in E$ the fact that $D^{\alpha} f(p)=0$ for some $p \in \Omega$ and all $\alpha \in \mathbb{N}_{0}^{n}$ implies that $f \equiv 0$ in the connected component of $\Omega$ that contains $p$.

In the case of Denjoy-Carleman classes, quasianalyticity is characterised by the following theorem.

Theorem 2.8 (Denjoy [9]-Carleman [6,7]) The space $\mathcal{E}_{\mathcal{M}}(\Omega)$ is quasianalytic if and only if

$$
\sum_{k=1}^{\infty} \frac{m_{k-1}}{k m_{k}}=\infty .
$$

We say that a weight sequence is quasianalytic if it satisfies (2.2) and nonquasianalytic if not.

Example 2.9 Let $\sigma>0$ be a parameter. We define a family $\mathcal{N}^{\sigma}$ of normal weight sequences by $n_{0}^{\sigma}=n_{1}^{\sigma}=1$ and

$$
n_{k}^{\sigma}=(\log (k+e))^{\sigma k}
$$


for $k \geq 2$. The weight sequence $\mathcal{N}^{\sigma}$ is quasianalytic if and only if $0<\sigma \leq 1$, see [35].

If $\mathcal{M}$ is a quasianalytic regular weight sequence then it is possible to show a quasianalytic version of Nagano's theorem [27], c.f. [14]. As in the case of the ultradifferentiable version of Sussmann's theorem, the proof is just a straightforward adaptation of the proof of the classical result, see e.g. [2].

Theorem 2.10 Let $U$ be an open neighbourhood of $p_{0} \in \mathbb{R}^{n}$ and $\mathcal{M}$ a quasianalytic regular weight sequence. Furthermore, let $\mathfrak{g}$ be a Lie subalgebra of $\mathfrak{X}_{\mathcal{M}}(U)$ that is also an $\mathcal{E}_{\mathcal{M}}$-module, i.e. if $X \in \mathfrak{g}$ and $f \in \mathcal{E}_{\mathcal{M}}(U)$ then $f X \in \mathfrak{g}$.

Then there exists an ultradifferentiable submanifold $W$ of class $\{\mathcal{M}\}$ in $U$, such that

$$
T_{p} W=\mathfrak{g}(p) \quad \forall p \in W
$$

Moreover, the germ of $W$ at $p_{0}$ is uniquely defined by this property.

As in the analytic category, c.f. [2], we have the following result.

Corollary 2.11 Let $\mathcal{M}$ be quasianalytic and $\mathfrak{D} \subseteq \mathfrak{X}_{\mathcal{M}}(\Omega)$ a collection of ultradifferentiable vector fields. If $\mathfrak{g}=\mathfrak{g}_{\mathfrak{D}}$ is the Lie algebra generated by $\mathfrak{D}$ and $p_{0} \in \Omega$ then the local Sussmann orbit of $p_{0}$, relative to $\mathfrak{D}$, coincides with the local Nagano leaf of $\mathfrak{g}$.

Proof Let $W_{N}$ be a representative of the local Nagano leaf of $\mathfrak{g}$ at $p_{0}$ and $W_{S}$ a representative of the local Sussmann orbit of $p_{0}$, relative to $\mathfrak{D}$. By Theorem 2.6 (1) there exists an open neighbourhood $V$ of $p_{0}$ such that $W_{S} \cap V \subseteq W_{N} \cap V$. On the other hand $\mathfrak{g}(p)=T_{p} W_{N}$ for all $p \in W_{N}$ and $\mathfrak{g}(p) \subseteq T_{p} W_{S}$ at every $p \in W_{S}$, hence $\mathfrak{g}(p)=T_{p} W_{S}$ for $p \in W_{S} \cap V$. The uniqueness part of Theorem 2.10 gives the equality of the local Nagano leaf and the local Sussmann orbit.

We want to close this section by showing how the results pertaining the division of smooth functions in [15, section 4] transfer to the category of ultradifferentiable functions of class $\{\mathcal{M}\}$. This is possible because these classes are closed under division by a coordinate, i.e. Lemma 2.4 holds.

Proposition 2.12 Let $U \subseteq \mathbb{R}^{n}$ be a neighbourhood of $0, \lambda \in \mathcal{E}_{\mathcal{M}}(U)$ and suppose that $\lambda$ is of the form $\lambda(x)=x^{\alpha} \tilde{\lambda}(x)$ where $\alpha \in \mathbb{N}_{0}^{n}$ and $\tilde{\lambda} \in \mathcal{E}_{\mathcal{M}}(U)$ with $\tilde{\lambda}(0) \neq 0$.

If $u$ is a locally integrable function near 0 with the property that the product $f:=\lambda \cdot u$ is of class $\{\mathcal{M}\}$ near the origin, then $u$ is also ultradifferentiable near 0.

Proof First, suppose that $\alpha=k e_{j}$ where $k \in \mathbb{N}$ and $e_{j}$ is the $j$-th unit vector. W.l.o.g. we may assume that $j=n$.

Using Lemma 2.4 we conclude that on some neighbourhood $V$ of the origin there is $f_{1} \in \mathcal{E}_{\mathcal{M}}(V)$ such that $f\left(x^{\prime}, x_{n}\right)=x_{n} f_{1}\left(x^{\prime}, x_{n}\right)$ on $V$. We want to show that $f_{1}\left(x^{\prime}, 0\right)=0$ for $\left(x^{\prime}, 0\right) \in V$ if $k>1$ : Suppose that there exists some $y \in \mathbb{R}^{n-1}$ with $(y, 0) \in V$ and $f_{1}(y, 0) \neq 0$. Then there is a neighbourhood $W$ of $(y, 0)$ such 
that $f_{1}(x) \neq 0$ and also $\tilde{\lambda}(x) \neq 0$ for $x \in W$. W.l.o.g. the open set $W$ is of the form $W=W^{\prime} \times I \subseteq \mathbb{R}^{n-1} \times \mathbb{R}$ and we set

$$
F\left(x_{n}\right):=\int_{W^{\prime}}\left|\frac{f_{1}}{\tilde{\lambda}}(x)\right| d x
$$

for $x_{n} \in I$. We conclude that

$$
\int_{W}|u(x)| d x=\int_{I}\left|x_{n}\right|^{1-k} F\left(x_{n}\right) d x=\infty
$$

and hence $u$ cannot be locally integrable near $(y, 0)$ which contradicts our assumption. Therefore, we obtain by iteration a function $\tilde{f}$ of class $\{\mathcal{M}\}$ defined near the origin in $\mathbb{R}^{n}$ such that $f\left(x^{\prime}, x_{n}\right)=x_{n}^{k} \tilde{f}\left(x^{\prime}, x_{n}\right)$. Hence $u=\tilde{f} / \tilde{\lambda}$ is also of class $\{\mathcal{M}\}$ in a neighbourhood of 0 .

In the general case, we argue as follows: Set $\tilde{f}=f / \tilde{\lambda}$ and

$$
u_{k}(x)=\prod_{j=k+1}^{n} x_{j}^{\alpha_{j}} u(x)
$$

for all $k \in\{1, \ldots, n-1\}$. The function $\tilde{f}$ is of class $\{\mathcal{M}\}$ whereas the functions $u_{k}$ are locally integrable near 0 . Furthermore, we define $u_{n}=u$ and obtain

$$
\begin{aligned}
x_{1}^{\alpha_{1}} u_{1}(x) & =\tilde{f}(x) \\
x_{k+1}^{\alpha_{k+1}} u_{k+1}(x) & =u_{k}(x) \quad 1 \leq k \leq n-1 .
\end{aligned}
$$

Hence repeated application of the first part finishes the proof.

\section{Almost-Analytic Extensions and the Wavefront Set in the Ultradifferentiable Setting}

In this section, we recall the almost-analytic extension of ultradifferentiable functions given by Dyn'kin in $[10,11]$ and the ultradifferentiable wavefront set introduced by Hörmander in [17]. The connection between the both was proven in [14].

We recall (see e.g. [36]) that a smooth function $F$ given on an open subset $\tilde{\Omega} \subseteq \mathbb{C}^{n}$ is almost analytic iff

$$
\bar{\partial}_{j} F=\frac{\partial}{\partial \bar{z}_{j}} F=\frac{1}{2}\left(\frac{\partial}{\partial x_{j}}+i \frac{\partial}{\partial y_{j}}\right) F
$$

is flat on $\tilde{\Omega} \cap \mathbb{R}^{n}$. The motivation to consider almost-analytic functions in the ultradifferentiable setting is the well-known fact that a function $f$ is smooth on $\Omega$ if and only if there is an almost-analytic function $F$ on some open set $\tilde{\Omega} \subseteq \mathbb{C}^{n}$ with $\tilde{\Omega} \cap \mathbb{R}^{n}=\Omega$ such that $\left.F\right|_{\Omega}=f$. In the ultradifferentiable category, the idea is now that if $f$ is ultradifferentiable of class $\{\mathcal{M}\}$ then it should be possible to construct an almost-analytic 
extension $F$ of $f$ such that the decrease of $\bar{\partial}_{j} F$ can be measured in terms of the weight sequence $\mathcal{M}$ (c.f. [12]).

In order to specify this decay, we introduce for a regular weight sequence $\mathcal{M}$ its associated weight given by

$$
h_{\mathcal{M}}(t)=\inf _{k} t^{k} m_{k} \quad \text { if } t>0 \quad \& \quad h_{\mathcal{M}}(0)=0 .
$$

Conversely, it is possible to extract the weight sequence from its weight, i.e.

$$
m_{k}=\sup _{t} \frac{h_{\mathcal{M}}(t)}{t^{k}} .
$$

The weight $h_{\mathcal{M}}$ is continuous with values in $[0,1]$, equals 1 on $[1, \infty)$ and goes more rapidly to 0 than $t^{p}$ for any $p>0$ for $t \rightarrow 0$, c.f. [14]. Recall also that if $K \subseteq \Omega$ is compact then $\mathcal{E}_{\mathcal{M}}(K)$ is the space of smooth functions which are defined on some neighbourhood of $K$ and on $K$ they satisfy (2.1) for some constants $C, h>0$, (c.f. [21]). We are now able to state the main result of [11].

Theorem 3.1 Let $\mathcal{M}$ be a regular weight sequence, $K \subset \subset \mathbb{R}^{n}$ a compact and convex set with $K=\overline{K^{\circ}}$. Then $f \in \mathcal{E}_{\mathcal{M}}(K)$ if and only if there exists a test function $F \in$ $\mathcal{D}\left(\mathbb{C}^{n}\right)$ with $\left.F\right|_{K}=f$ and

$$
\left|\bar{\partial}_{j} F(z, \bar{z})\right| \leq C h_{\mathcal{M}}\left(Q d_{K}(z)\right)
$$

where $1 \leq j \leq n$ and $d_{K}$ is the distance function with respect to $K$ on $\mathbb{C}^{n} \backslash K$.

We call such function $F$ an $\mathcal{M}$-almost-analytic extension of $f$.

The following theorem is the $\mathcal{M}$-almost-analytic version of the almost-holomorphic implicit function theorem proven in [24]. The proof is virtually the same as in the smooth case.

Theorem 3.2 Let $\mathcal{M}$ be a regular weight sequence, $U \subseteq \mathbb{C}^{N}$ a neighbourhood of the origin, $A \in \mathbb{C}^{p}$ and $F: U \times \mathbb{C}^{p} \rightarrow \mathbb{C}^{N}$ of class $\{\mathcal{M}\}$ on $U$ and polynomial in the last variable with $F(0, A)=0$ and $F_{Z}(0, A)$ is invertible. Then there exists a neighbourhood $U^{\prime} \times V^{\prime}$ of $(0, A)$ and a smooth mapping $\phi=\left(\phi_{1}, \ldots, \phi_{N}\right)$ : $U^{\prime} \times V^{\prime} \rightarrow \mathbb{C}^{N}$ with $\phi(0, A)=0$ with the property that if $F(Z, \bar{Z}, W)=0$ for some $(Z, W) \in U^{\prime} \times V^{\prime}$ then $Z=\phi(Z, \bar{Z}, W)$. Furthermore, there are constants $C, \gamma>0$ such that

$$
\left|\frac{\partial \phi_{j}}{\partial Z_{k}}(Z, \bar{Z}, W)\right| \leq C h_{\mathcal{M}}(\gamma|\phi(Z, \bar{Z}, W)-Z|)
$$

for all $1 \leq j, k \leq N$ and $\phi$ is holomorphic in $W$.

In the following, we recall the results on the ultradifferentiable wavefront set that we need in this paper. We start with the definition given in [17].

Definition 3.3 Let $\mathcal{M}$ be a regular weight sequence, $u \in \mathcal{D}^{\prime}(\Omega)$ and $\left(x_{0}, \xi_{0}\right) \in T^{*} \Omega \backslash$ $\{0\}$. We say that $u$ is microlocally ultradifferentiable of class $\{\mathcal{M}\}$ at $\left(x_{0}, \xi_{0}\right)$ iff there is 
a bounded sequence $\left(u_{N}\right)_{N} \subseteq \mathcal{E}^{\prime}(\Omega)$ such that $\left.\left.u_{N}\right|_{V} \equiv u\right|_{V}$, where $V$ is a neighbourhood of $\left(x_{0}\right)$, and a conic neighbourhood $\Gamma$ of $\xi_{0}$ such that for some constant $Q>0$

$$
\sup _{\substack{\xi \in \Gamma \\ N \in \mathbb{N}_{0}}} \frac{|\xi|^{N}\left|\hat{u}_{N}\right|}{Q^{N} m_{N} N !}<\infty .
$$

The ultradifferentiable wavefront set $\mathrm{WF}_{\mathcal{M}} u$ is then defined as

$$
\mathrm{WF}_{\mathcal{M}} u:=\left\{(x, \xi) \in T^{*} \Omega \backslash\{0\} \mid u \text { is not microlocally ultradiff. of class }\{\mathcal{M}\} \text { at }(x, \xi)\right\} \text {. }
$$

We refer to [19] for the basic properties of $\mathrm{WF}_{\mathcal{M}} u$. We shall just mention three facts, that turn out to be important later on. First, if $(p, \xi) \notin \mathrm{WF}_{\mathcal{M}} u$ for all $\xi \in \mathbb{R}^{n} \backslash\{0\}$ then $u$ is of class $\{\mathcal{M}\}$ near $p$. If $P=\sum p_{\alpha} D^{\alpha}$ is a linear partial differential operator with $\mathcal{E}_{\mathcal{M}}$-coefficients, then

$$
\mathrm{WF}_{\mathcal{M}} P u \subseteq \mathrm{WF}_{\mathcal{M}} u
$$

Additionally, we note that $\mathrm{WF}_{\mathcal{M}} u$ satisfies the following microlocal reflection property:

$$
(x, \xi) \notin \mathrm{WF}_{\mathcal{M}} u \Longleftrightarrow(x,-\xi) \notin \mathrm{WF}_{\mathcal{M}} \bar{u} .
$$

In particular, if $u$ is a real-valued distribution, i.e. $\bar{u}=u$, then $\left.\operatorname{WF}_{\mathcal{M}} u\right|_{x}:=\left\{\xi \in \mathbb{R}^{n} \mid\right.$ $\left.(x, \xi) \in \mathrm{WF}_{\mathcal{M}} u\right\}$ is symmetric at the origin.

It is a classic fact that the analytic wavefront set can not only be characterised by the Fourier transform but also by holomorphic extensions in certain directions, see [5]. Likewise, the smooth wavefront set can be characterised by almost-analytic extensions, c.f. [26].

In [14], we showed that the ultradifferentiable wavefront set can be characterised by $\mathcal{M}$-almost-analytic extensions. This fact in turn allowed us to show that $\mathrm{WF}_{\mathcal{M}} u$ can be invariantly defined for distributions on manifolds of class $\{\mathcal{M}\}$.

In the remainder of this section, we want to recall two theorems from [14], which are crucial for the proof of Theorem 1.1. In order to state the first theorem, we need to recall some notations from [14], which will be also used in the proof of Theorem 1.1.

A subset $\Gamma \subseteq \mathbb{R}^{d}$ is a cone iff for all $\lambda>0$ and $y \in \Gamma$, we have $\lambda y \in \Gamma$. If $r>0$ then

$$
\Gamma_{r}:=\{y \in \Gamma|| y \mid<r\} .
$$

If $\Gamma^{\prime} \subseteq \Gamma$ is also a cone, we write $\Gamma^{\prime} \subset \subset \Gamma$ iff $\left(\Gamma^{\prime} \cap S^{d-1}\right) \subset \subset\left(\Gamma \cap S^{d-1}\right)$.

Inspired by [26, section 2.1] (c.f. also [24, section 2]) in the smooth category, we introduce the following notation. If $\mathcal{M}$ is a regular weight sequence with associated weight $h_{\mathcal{M}}$, then a function $F \in \mathcal{E}\left(\Omega \times U \times \Gamma_{r}\right), U \subseteq \mathbb{R}^{d}$ open is said to be $\mathcal{M}$ almost- analytic in the variables $(x, y) \in U \times \Gamma_{r}$ with parameter $x^{\prime} \in \Omega$ iff for all $K \subset \subset \Omega, L \subset \subset U$ and cones $\Gamma^{\prime} \subset \subset \Gamma$ there are constants $C, Q>0$ such that for some $r^{\prime}$ we have 


$$
\left|\frac{\partial F}{\partial \bar{z}_{j}}\left(x^{\prime}, x, y\right)\right| \leq C h_{\mathcal{M}}(Q|y|) \quad\left(x^{\prime}, x, y\right) \in K \times L \times \Gamma_{r^{\prime}}^{\prime}, j=1, \ldots, d \text {. }
$$

We may also say generally that a function $g \in \mathcal{C}\left(\Omega \times U \times \Gamma_{r}\right)$ is of slow growth in $y \in \Gamma_{r}$ if for all $K \subset \subset \Omega, L \subset \subset U$ and $\Gamma^{\prime} \subset \subset \Gamma$ there are constants $c, k>0$ such that

$$
\left|g\left(x^{\prime}, x, y\right)\right| \leq c|y|^{-k} \quad\left(x^{\prime}, x, y\right) \in K \times L \times \Gamma_{r}^{\prime} .
$$

Theorem 3.4 Let $F \in \mathcal{E}\left(\Omega \times U \times \Gamma_{r}\right)$ be $\mathcal{M}$-almost analytic in the variables $(x, y) \in$ $U \times \Gamma_{r}$ and of slow growth in the variable $y \in \Gamma_{r}$. Then the distributional limit $u$ of the sequence $u_{\varepsilon}=F(., ., \varepsilon) \in \mathcal{E}(\Omega \times U)$ exists. We say that $u=b_{\Gamma}(F) \in \mathcal{D}^{\prime}(\Omega \times U)$ is the boundary value of $F$. Furthermore, we have

$$
\mathrm{WF}_{\mathcal{M}} u \subseteq(\Omega \times U) \times\left(\mathbb{R}^{n} \times \Gamma^{\circ}\right)
$$

where $\Gamma^{\circ}=\left\{\eta \in \mathbb{R}^{d} \mid\langle y, \eta\rangle \geq 0 \forall y \in \Gamma\right\}$ is the dual cone of $\Gamma$ in $\mathbb{R}^{d}$.

We close this section by recalling the last fact that we need from [14], the elliptic regularity theorem for partial differential operators with ultradifferentiable coefficients. More precisely we formulate the theorem for differential operators of class $\{\mathcal{M}\}$ acting on distributions with values in ultradifferentiable vector bundles. We refer to [8] for more details. We recall here just two details for the convenience of the reader. If $u$ is a distribution on an ultradifferentiable manifold $M$ of class $\{\mathcal{M}\}$ with values in an $\mathcal{E}_{\mathcal{M}}$-vector bundle over $M$, then we can write locally $\left.u\right|_{V}=\sum_{j=1}^{N} u_{j} \omega^{j}$, where the sections $\omega^{1}, \ldots, \omega^{N} \in \mathcal{E}_{\mathcal{M}}\left(V,\left.E\right|_{V}\right)$ constitute a local basis of $\mathcal{E}_{\mathcal{M}}(M, E)$. The ultradifferentiable wavefront set of $u$ is then defined locally by

$$
\mathrm{WF}_{\mathcal{M}} u=\bigcup_{j=1}^{N} \mathrm{WF}_{\mathcal{M}} u_{j}
$$

On the other hand, if $M$ is an manifold of class $\{\mathcal{M}\}, E$ and $F$ are two $\mathcal{E}_{\mathcal{M}^{-}}$ vector bundles with the same fibre dimension and $P: \mathcal{E}_{\mathcal{M}}(M, E) \rightarrow \mathcal{E}_{\mathcal{M}}(M, F)$ is a partial differential operator of class $\{\mathcal{M}\}$ then the principal symbol $p$ of $P$ is an ultradifferentiable mapping on $T^{*} M$ with values in the fibre-linear maps from $E$ to $F$. The operator $P$ is said to be not characteristic (or noncharacteristic) at a point $(x, \xi) \in T^{*} M \backslash\{0\}$ if $p(x, \xi)$ is an invertible linear mapping. The set of all characteristic points is defined by

$$
\text { Char } P=\left\{(x, \xi) \in T^{*} M \backslash\{0\}: P \text { is characteristic at }(x, \xi)\right\} \text {. }
$$

Theorem 3.5 Let $\mathcal{M}$ be a normal weight sequence, $M$ an ultradifferentiable manifold of class $\{\mathcal{M}\}$ and $E, F$ two ultradifferentiable vector bundles on $M$ of the same fibre dimension. If $P(x, D)$ is a partial differential operator of class $\{\mathcal{M}\}$ between $E$ and $F$ then 


$$
\mathrm{WF}_{\mathcal{M}} u \subseteq \mathrm{WF}_{\mathcal{M}}(P u) \cup \operatorname{Char} P \quad u \in \mathcal{D}^{\prime}(M, E)
$$

\section{CR Manifolds of Denjoy Carleman Type}

Recall that an abstract CR manifold $(M, \mathcal{V})$ is a smooth manifold $M$ with a subbundle $\mathcal{V} \subseteq \mathbb{C} T M$, called the CR bundle, that is formally integrable, i.e. $[\mathcal{V}, \mathcal{V}] \subseteq \mathcal{V}$ and such that $\mathcal{V} \cap \overline{\mathcal{V}}=\{0\}$. The $\mathrm{CR}$ dimension of $M$ is $\operatorname{dim}_{\mathbb{C}} \mathcal{V}$. Important examples of $\mathrm{CR}$ manifolds are generic submanifolds of $\mathbb{C}^{N}$. For a generic submanifold $M \subseteq \mathbb{C}^{N}$ the CR bundle $\mathcal{V}$ is given by $\mathcal{V}=T^{0,1} \mathbb{C}^{N} \cap \mathbb{C} T M$, where $T^{0,1} \mathbb{C}^{N}$ is the complex bundle generated by the vector fields $\partial / \partial \bar{z}_{j}$. We refer to [2] for a detailed account of the theory of CR manifolds. Here we just summarise the notations and basic facts that we need in the following.

The sections of $\mathcal{V}$ are the $\mathrm{CR}$ vector fields of $M$. A CR function (or CR distribution) is a function (or distribution) on $M$ that is annihilated by all CR vector fields. We refer to $T^{\prime} M:=\mathcal{V}^{\perp}$ as the holomorphic cotangent bundle and to its sections as holomorphic forms. The real subbundle $T^{0} M \subseteq T^{\prime} M$ that consists of the real dual vectors that vanish on $\mathcal{V} \oplus \overline{\mathcal{V}}$ is called the characteristic bundle of $M$ and its sections are the characteristic forms on $M$. Note that if $L$ is a CR vector field, we have generally that Char $L \subseteq T^{0} M$.

A $\mathcal{C}^{1}$-mapping $H$ between two CR manifolds $(M, \mathcal{V})$ and $\left(M^{\prime}, \mathcal{V}^{\prime}\right)$ is CR iff for all $p \in M$ we have $H_{*}\left(\mathcal{V}_{p}\right) \subseteq \mathcal{V}_{H(p)}^{\prime}$. Here $H_{*}$ denotes the tangent map of $H$. If $M^{\prime} \subseteq \mathbb{C}^{N^{\prime}}$ is an embedded CR submanifold and $Z^{\prime}=\left(Z_{1}^{\prime}, \ldots, Z_{N^{\prime}}^{\prime}\right)$ some set of local holomorphic coordinates in $\mathbb{C}^{N^{\prime}}$ then $H_{j}=Z_{j}^{\prime} \circ H, 1 \leq j \leq N^{\prime}$ is a $\mathrm{CR}$ function on the CR manifold $M$ for all $1 \leq j \leq N^{\prime}$.

We note that, as in the smooth case, c.f. [2], we can choose coordinates such that the defining equation of a generic submanifold is of a special form.

Proposition 4.1 Let $M \subseteq \mathbb{C}^{N}$ be a generic manifold of class $\{\mathcal{M}\}$ of codimension $d$ and $p_{0} \in M$. If $n$ denotes the $C R$ dimension of $M$ then there are holomorphic coordinates $(z, w) \in \mathbb{C}^{n} \times \mathbb{C}^{d}$ defined near $p_{0}$ that vanish at $p_{0}$ and a function $\varphi \in \mathcal{E}_{\mathcal{M}}\left(U \times V, \mathbb{R}^{d}\right)$ defined on a neighbourhood $U \times V$ of the origin in $\mathbb{R}^{2 n} \times \mathbb{R}^{d}$ with $\varphi(0)=0$ and $\nabla \varphi(0)=0$, such that near $p_{0}$ the manifold $M$ is given by

$$
\operatorname{Im} w=\varphi(z, \bar{z}, \operatorname{Re} w) .
$$

The proof of Proposition 4.1 is analogous to the proof of the statement in the smooth category, [2, Theorem 1.3.6] since the implicit function theorem holds in regular Denjoy-Carleman classes, c.f. [4].

Next we give a first result on the structure of ultradifferentiable CR manifolds. For that we have to recall some further definitions from [2]. Let $M \subseteq \mathbb{C}^{N}$ be a CR submanifold of class $\{\mathcal{M}\}$ and $p \in M$. The CR orbit Orb $p$ of $p$ is the local Sussmann orbit of $p$ in $M$ relative to the set of ultradifferentiable sections of $T^{c} M=\operatorname{Re} \mathcal{V}$. Note that if $p \in M$ then by construction $T_{q}^{c} \operatorname{Orb}_{q}=T_{p}^{c} M$ for all $Q \in \operatorname{Orb}_{p_{0}}$ thence $\operatorname{Orb}_{p}$ is the germ of a CR submanifold of $\mathbb{C}^{N}$ with CR dimension $n$. 
We say that $M$ is minimal at $p_{0}$ iff there is no submanifold $S \subseteq M$ through $p_{0}$ such that $T_{p}^{c} M \subseteq T_{p} S$ for all $p \in S$ and $\operatorname{dim}_{\mathbb{R}} S<\operatorname{dim}_{\mathbb{R}} M$. The manifold $M$ is said to be of finite type at $p_{0}$ iff there are vector fields $X_{1}, \ldots, X_{k} \in \mathcal{E}_{\mathcal{M}}\left(M, T^{c} M\right)$ such that the Lie algebra generated by the $X_{1}, \ldots, X_{k}$ evaluated at $p_{0}$ is isomorphic to $T_{p_{0}} M$. It is well known that finite type implies minimality and that the two notions coincide for real-analytic CR manifolds, c.f. [2]. We are going to show that this fact holds also for quasianalytic CR submanifolds.

Theorem 4.2 Let $\mathcal{M}$ be a quasianalytic regular weight sequence and $M \subseteq \mathbb{C}^{N}$ an ultradifferentiable CR manifold of class $\{\mathcal{M}\}$. The following statements are equivalent:

(1) $M$ is minimal at $p_{0}$.

(2) $\operatorname{dim}_{\mathbb{R}} \operatorname{Orb}_{p_{0}}=\operatorname{dim}_{\mathbb{R}} M$.

(3) $M$ is of finite type at $p_{0}$.

Proof The equivalence of (1) and (2) holds even if $\mathcal{M}$ is nonquasianalytic. Following the arguments in $[2, \S 4.1$.] we see that, if we assume that $M$ is nonminimal then $\operatorname{dim}_{\mathbb{R}} \operatorname{Orb}_{p_{0}}<\operatorname{dim}_{\mathbb{R}} M$. On the other hand, if $\operatorname{dim}_{\mathbb{R}} \operatorname{Orb}_{p_{0}}<\operatorname{dim}_{\mathbb{R}} M$ then any representative $W$ of $\operatorname{Orb}_{p_{0}}$ is by the remark above a proper submanifold of $M$ and $T_{p}^{c} W=T_{p}^{c} M$ for all $p \in W$. It remains to prove that (2) implies (3).

By Corollary 2.11, we have that $\operatorname{Orb}_{p_{0}}=\gamma_{p_{0}}(\mathfrak{g})$, where $\mathfrak{g}$ is the Lie algebra generated by the ultradifferentiable sections of $T^{c} U$ with $U$ being a sufficiently small neighbourhood of $p_{0}$ and $\gamma_{p_{0}}(\mathfrak{g})$ the local Nagano leaf of $\mathfrak{g}$ at $p_{0}$. Hence $\operatorname{dim}_{\mathbb{R}} \operatorname{Orb}_{p_{0}}=$ $\operatorname{dim}_{\mathbb{R}} \gamma_{p_{0}}(\mathfrak{g})=\operatorname{dim}_{\mathbb{R}} \mathfrak{g}\left(p_{0}\right)$.

On the other hand, $M$ is of finite type at $p_{0}$ if and only if $\operatorname{dim}_{\mathbb{R}} \mathfrak{g}\left(p_{0}\right)=\operatorname{dim}_{\mathbb{R}} M$.

The next example is a straightforward generalisation of [2, Example 1.5.16].

Example 4.3 Let $\mathcal{M}$ be a nonquasianalytic regular weight sequence and $\psi \in \mathcal{E}_{\mathcal{M}}(\mathbb{R})$ a real-valued function such that $\psi(y)=0$ for $y \leq 0$ and $\psi(y)>0$ for $y>0$. We define a real hypersurface in $\mathbb{C}^{2}$ by

$$
M=\left\{(z, w) \in \mathbb{C}^{2} \mid \operatorname{Im} w=\varphi(\operatorname{Im} z)\right\}
$$

Then $M$ is minimal at the origin but not of finite type at 0 . Indeed, if $M$ is nonminimal at 0 then according to [2, Theorem 1.5.15] there is a holomorphic hypersurface $S \subseteq M$ through the origin. Since $\partial / \partial z$ is tangent to $S$ at 0 it follows that $S$ is given near the origin by the defining equation $w=h(z)$ where $h$ is a holomorphic function defined in some neighbourhood of $0 \in \mathbb{C}$ with $h(0)=0$. We conclude that due to $S \subseteq M$ we necessarily have that

$$
h(z)-\overline{h(z)}=2 i \psi(\operatorname{Re} z)
$$

in some neighbourhood of 0 . It follows that $\psi$ has to be real analytic near 0 which contradicts the definition of $\psi$. Since $\psi$ is flat at the origin, it follows that $M$ cannot be of finite type at 0 . 
We close this section by recalling the space of multipliers for an ultradifferentiable abstract CR manifold $(M, \mathcal{V})$, which was introduced in [15] for the smooth setting. To begin with, consider the following sequence of spaces of sections

$$
E_{k}=\left\langle\mathcal{L}_{K_{1}} \ldots \mathcal{L}_{K_{j}} \theta: j \leq k, \quad K_{q} \in \mathcal{E}_{\mathcal{M}}(M, \mathcal{V}), \theta \in \mathcal{E}_{\mathcal{M}}\left(M, T^{0} M\right)\right\rangle
$$

We note that $E_{0}=\mathcal{E}_{\mathcal{M}}\left(M, T^{0} M\right)$, and $E_{j} \subseteq \mathcal{E}_{\mathcal{M}}\left(M, T^{\prime} M\right)$ for all $j \in \mathbb{N}_{0}$, and set $E=\bigcup_{j \in \mathbb{N}_{0}} E_{j}$.

We associate to the increasing chain $E_{k}$ the increasing sequence of ideals $\mathcal{S}^{k} \subset$ $\mathcal{E}_{\mathcal{M}}(M, \mathbb{C})$, where

$$
\mathcal{S}^{k}=\bigwedge^{N} E_{k}=\left\{\operatorname{det}\left(\begin{array}{ccc}
V^{1}\left(\mathfrak{Y}_{1}\right) & \ldots & V^{1}\left(\mathfrak{Y}_{N}\right) \\
\vdots & & \vdots \\
V^{N}\left(\mathfrak{Y}_{1}\right) & \ldots & V^{N}\left(\mathfrak{Y}_{N}\right)
\end{array}\right): V^{j} \in E_{k}, \mathfrak{Y}_{j} \in \mathcal{E}_{\mathcal{M}}\left(M,\left(T^{\prime} M\right)^{*}\right)\right\} .
$$

We set $\mathcal{S}=\mathcal{S}(M)=\bigcup_{k \in \mathbb{N}_{0}} \mathcal{S}^{k}$ and call it the space of multipliers of $M$. In fact each $\mathcal{S}^{k}$ and thus also $\mathcal{S}$ can be considered actually as ideal sheaves, if we define $E^{k}(U)$ and $\mathcal{S}^{k}(U)$ accordingly.

Note that locally one can find smaller sets of generators: Let $U \subseteq M$ be open, and assume that $L_{1}, \ldots, L_{n}$ is a local basis for $\Gamma(U, \mathcal{V})$, that $\theta^{1}, \ldots, \theta^{d}$ is a local basis for $\Gamma\left(U, T^{0} M\right)$, and that $\omega^{1}, \ldots, \omega^{N}$ is a local basis of $T^{\prime} M$. We write $\mathcal{L}_{j}=\mathcal{L}_{L_{j}}$ for $j=1, \ldots, n$ and $\mathcal{L}^{\alpha}=\mathcal{L}_{1}^{\alpha_{1}} \ldots \mathcal{L}_{n}^{\alpha_{n}}$ for any multi-index $\alpha=\left(\alpha_{1}, \ldots, \alpha_{n}\right) \in \mathbb{N}^{n}$. We note that, since $\mathcal{V}$ is formally integrable, the $\mathcal{L}^{\alpha}$, where $|\alpha|=k$, generate all $k$-th-order homogeneous differential operators in the $\mathcal{L}_{j}$, and we thus have

$$
\left.E_{k}\right|_{U}=\left\langle\mathcal{L}^{\alpha} \theta^{\mu}: \quad 1 \leq \mu \leq d,|\alpha| \leq k\right\rangle .
$$

We can expand

$$
\mathcal{L}^{\alpha} \theta^{\mu}=\sum_{\ell=1}^{N} A_{\ell}^{\alpha, \mu} \omega^{\ell}
$$

and for any choice $\underline{\alpha}=\left(\alpha^{1}, \ldots, \alpha^{N}\right)$ of multi-indices $\alpha^{1}, \ldots, \alpha^{N} \in \mathbb{N}_{0}^{n}$ and $r=$ $\left(r_{1}, \ldots, r_{N}\right) \in\{1, \ldots, d\}^{N}$ we define the functions

$$
D(\underline{\alpha}, r)=\operatorname{det}\left(\begin{array}{ccc}
A_{1}^{\alpha^{1}, r_{1}} & \ldots & A_{N}^{\alpha^{1}, r_{1}} \\
\vdots & & \vdots \\
A_{1}^{\alpha^{N}, r_{N}} & \ldots & A_{N}^{\alpha^{N}, r_{N}}
\end{array}\right)
$$

With this notation, we have

$$
\left.\mathcal{S}^{k}\right|_{U}=\left\langle D(\underline{\alpha}, r):\left|\alpha^{j}\right| \leq k\right\rangle ;
$$


we shall denote the stalk of $\mathcal{S}^{k}$ at $p$ by $\mathcal{S}_{p}^{k}$.

The space of multipliers of a CR manifold $M$ clearly encodes the nondegeneracy properties of $M$. We close this section by taking a closer look at the connection of $\mathcal{S}$ with finite nondegeneracy. We recall from [2] the definition of finite nondegeneracy for abstract CR manifolds.

Definition 4.4 Let $M$ be an abstract CR manifold and

$$
E_{k}(p)=\left\langle\mathcal{L}_{K_{1}} \ldots \mathcal{L}_{K_{j}} \theta(p): j \leq k, \quad K_{q} \in \mathcal{E}(M, \mathcal{V}), \theta \in \mathcal{E}\left(M, T^{0} M\right)\right\rangle
$$

for $p \in M$ and $k \in \mathbb{N}$. Then $M$ is $k_{0}$-nondegenerate at $p_{0} \in M$ iff $E_{k_{0}-1} \subsetneq E_{k_{0}}=$ $T_{p_{0}}^{\prime} M$. We say that $M$ is finite nondegenerate iff $M$ is finite nondegenerate at every point.

Remark 4.5 This definition is in fact local, since by [2, Proposition 11.1.10], if $L_{1}, \ldots, L_{n}$ is a local basis of CR vector fields and $\theta^{1}, \ldots \theta^{d}$ is a local basis of characteristic forms near $p_{0}$ then $M$ is $k_{0}$-nondegenerate if and only if

$$
T_{p_{0}}^{\prime} M=\operatorname{span}_{\mathbb{C}}\left\{\mathcal{L}^{\alpha} \theta^{\mu}\left(p_{0}\right):|\alpha| \leq k_{0}, \mu \in\{1, \ldots, d\}\right\}
$$

Hence we may replace $M$ with any open neighbourhood $U \subseteq M$ of $p_{0}$ in (4.4). Thus we observe that a CR submanifold $M$ is $k_{0}$-nondegenerate at $p_{0} \in M$ if and only if $\mathcal{S}_{p_{0}}^{k_{0}}=\left(\mathcal{E}_{\mathcal{M}}\right)_{p_{0}}$.

More precisely, let $U \subseteq M$ be an open subset and $q \in U$. Then $M$ is $k_{0^{-}}$ nondegenerate at $q$ if and only if there is a multiplier $f \in \mathcal{S}^{k_{0}}(U)$ that does not vanish at $q$, i.e. $f(q) \neq 0$. Indeed, if $f(q) \neq 0$ then obviously $E_{k_{0}}(q)=T_{q}^{\prime} M$. On the other hand, if $g(q)=0$ for all multipliers $g \in \mathcal{S}^{k_{0}}(U)$ then necessarily $E_{k_{0}}(q) \neq T_{q}^{\prime} M$.

\section{Ultradifferentiable Regularity of CR Mappings}

The main goal of this section is to present the proof of Theorem 1.1. Furthermore, we show also ultradifferentiable versions of further regularity results of [3] and [24]. However, first we need to recall the definition of finite nondegeneracy of a CR mapping.

Definition 5.1 Let $M$ be an abstract CR manifold and $M^{\prime} \subseteq \mathbb{C}^{N^{\prime}}$ a generic submanifold. Furthermore let $\rho^{\prime}=\left(\rho_{1}^{\prime}, \ldots, \rho_{d^{\prime}}^{\prime}\right)$ be a defining function of $M^{\prime}$ near a point $q_{0} \in M^{\prime}, L_{1}, \ldots, L_{n}$ a local basis of CR vector fields on $M$ near $p_{0} \in M$ and $H: M \rightarrow M^{\prime}$ a $C^{m}$-CR mapping with $H\left(p_{0}\right)=q_{0}$.

For $0 \leq k \leq m$ define an increasing sequence of subspaces $E_{k}\left(p_{0}\right) \subseteq \mathbb{C}^{N^{\prime}}$ by

$$
E_{k}\left(p_{0}\right):=\operatorname{span}_{\mathbb{C}}\left\{\left.L^{\alpha} \frac{\partial \rho^{\prime}}{\partial Z^{\prime}}(H(Z), \overline{H(Z)})\right|_{Z=p_{0}}: 0 \leq|\alpha| \leq k, 1 \leq l \leq d^{\prime}\right\}
$$

We say that $H$ is $k_{0}$-nondegenerate at $p_{0}\left(0 \leq k_{0} \leq m\right)$ iff $E_{k_{0}-1}\left(p_{0}\right) \subsetneq E_{k_{0}}\left(p_{0}\right)=$ $\mathbb{C}^{N^{\prime}}$. 
Remark 5.2 Comparing Definition 5.1 with Definition 4.4 we observe that a CR submanifold $M \in \mathbb{C}^{N}$ is $k_{0}$-nondegenerate if and only if id : $M \rightarrow M$ is $k_{0^{-}}$ nondegenerate. We note also the fact that any CR diffeomorphism between two $k_{0}$-nondegenerate $\mathrm{CR}$ submanifolds is $k_{0}$-nondegenerate.

Finally, we need to recall that if $\rho$ is a local defining function of $M, \Gamma \subseteq \mathbb{R}^{d}$ an open convex cone, $p_{0} \in M$ and $U \subseteq \mathbb{C}^{N}$ an open neighbourhood of $p_{0}$, then a wedge $\mathcal{W}$ with edge $M$ centred at $p_{0}$ is an open subset of the form $\mathcal{W}:=\{Z \in U \mid \rho(Z, \bar{Z}) \in \Gamma\}$.

Proof of Theorem 1.1 Since the assertion of the theorem is local, we are going to work on a neighbourhood $\Omega \subseteq \mathbb{C}^{N}$ of $p_{0}$. If $\Omega$ is small enough then by Proposition 4.1 there are open neighbourhoods $U \subseteq \mathbb{C}^{n}$ and $V \subseteq \mathbb{R}^{d}$ of the origin and a function $\varphi \in \mathcal{E}_{\mathcal{M}}\left(U \times V, \mathbb{R}^{d}\right)$ with $\varphi(0,0)=0$ and $\nabla \varphi(0,0)=0$ such that

$$
M \cap \Omega=\{(z, w) \in \Omega \mid \operatorname{Im} w=\varphi(z, \bar{z}, \operatorname{Re} w)\} .
$$

From now on we denote $M \cap \Omega$ by $M$. If we choose $U$ and $V$ to be small enough, we can consider the diffeomorphism

$$
\begin{aligned}
\Psi: U \times V & \longrightarrow M \\
(z, s) & \longmapsto(z, s+i \varphi(z, \bar{z}, s)) .
\end{aligned}
$$

If we shrink the neighbourhoods $U, V$ a little bit (such that $\varphi \in \mathcal{E}_{\mathcal{M}}\left(\overline{U \times V}, \mathbb{R}^{d}\right)$ ) and assume that w.l.o.g. both sets are convex then by Theorem 3.1 we can extend the mapping $\Psi \mathcal{M}$-almost analytically in the $s$-variables, i.e. there exists a smooth function $\tilde{\Psi}: U \times V \times \mathbb{R}^{d} \rightarrow \mathbb{C}^{N}$ such that $\left.\tilde{\Psi}\right|_{U \times V \times\{0\}}=\Psi$ and for each component $\tilde{\Psi}_{k}, k=1, \ldots, N$, of $\tilde{\Psi}$ we have

$$
\left|\frac{\partial \tilde{\Psi}_{k}}{\partial \bar{w}_{j}^{\prime}}(z, \bar{z}, s, t)\right| \leq C h_{\mathcal{M}}(\gamma|t|) \quad j=1, \ldots, d,
$$

for some constants $C, \gamma>0$. Here $w^{\prime}=s+i t \in V+i \mathbb{R}^{d}$. We see that there is some $r>0$ such that $\left.\tilde{\Psi}\right|_{U \times V \times B_{r}(0)}$ is a diffeomorphism.

By assumption $H=\left(H_{1}, \ldots, H_{N^{\prime}}\right)$ extends continuously to a holomorphic mapping on a wedge $\mathcal{W}$ near 0 . If we shrink $\mathcal{W}$ we may assume that $\partial H_{j}, j=1, \ldots, N^{\prime}$, is bounded on $\mathcal{W}$. By definition

$$
\mathcal{W}=\left\{Z \in \Omega_{0} \mid \rho(Z, \bar{Z}) \in \tilde{\Gamma}\right\}
$$

for a neighbourhood $\Omega_{0}$ of the origin in $\mathbb{C}^{N}$ and an open acute cone $\tilde{\Gamma} \subseteq \mathbb{R}^{d}$. If we shrink $U, V$, when necessary, and choose a suitable open and acute cone $\Gamma$, we achieve that

$$
\tilde{\Psi}\left(U \times V \times \Gamma_{\delta}\right) \subseteq \mathcal{W}
$$

for some $r \geq \delta>0$. Note that $\tilde{\Psi}\left(U \times V \times \Gamma_{\delta}\right)$ is open in $\mathbb{C}^{N}$. For each $j=1, \ldots, N^{\prime}$ set $h_{j}=H_{j} \circ \tilde{\Psi}$ and $u_{j}=H_{j} \circ \Psi$. Since 


$$
\frac{\partial h_{j}}{\partial \bar{w}_{k}^{\prime}}=\sum_{\ell=1}^{N} \frac{\partial H_{j}}{\partial Z_{\ell}} \frac{\partial \tilde{\Psi}_{\ell}}{\partial \bar{w}_{k}^{\prime}} \quad j=1, \ldots, N^{\prime}, k=1, \ldots, d,
$$

and $\partial H_{j}$ is bounded, each function $h_{j}$ is $\mathcal{M}$-almost analytic on $U \times V \times \Gamma_{\delta}$ due to (5.1) and extends $u_{j} \in \mathcal{C}^{k_{0}}(U \times V)$. Hence Theorem 3.4 implies that

$$
\mathrm{WF}_{\mathcal{M}} u_{j} \subseteq(U \times V) \times\left(\mathbb{R}^{2 n} \times \Gamma^{\circ}\right) \backslash\{0\} .
$$

If $L_{j} \in \mathcal{E}_{\mathcal{M}}(M, \mathcal{V}), j=1, \ldots, n$, form a basis of the CR vector fields on $M=$ $M \cap \Omega$, then the vector fields $\Lambda_{j}=\Psi^{*} L_{j}$ define a CR structure on $U \times V$ and $\Lambda_{j} u_{k}=0$ for $j=1, \ldots, n$ and $k=1, \ldots, N^{\prime}$.

Let $\rho^{\prime}$ be a defining function of $M^{\prime}$ near $p_{0}^{\prime}=0 \in \mathbb{C}^{N^{\prime}}$. Then there are ultradifferentiable functions $\Phi_{\ell, \alpha}\left(Z^{\prime}, \bar{Z}^{\prime}, W\right)$ for $|\alpha| \leq k_{0}, \ell=1, \ldots, d^{\prime}$, defined in a neighbourhood of $\{0\} \times \mathbb{C}^{K_{0}} \subseteq \mathbb{C}^{N^{\prime}} \times \mathbb{C}^{K_{0}}$ and polynomial in the last $K_{0}=N^{\prime} \cdot \mid\{\alpha \in$ $\left.\mathbb{N}_{0}^{n}|| \alpha \mid \leq k_{0}\right\} \mid$ variables such that

$$
\Lambda^{\alpha}\left(\rho_{\ell}^{\prime} \circ u\right)(z, \bar{z}, s)=\Phi_{\ell, \alpha}\left(u(z, \bar{z}, s), \bar{u}(z, \bar{z}, s),\left(\Lambda^{\beta} \bar{u}(z, \bar{z}, s)\right)_{|\beta| \leq k_{0}}\right)=0
$$

and

$$
\Lambda^{\alpha} \rho_{\ell, Z^{\prime}}^{\prime}(u, \bar{u})(0,0,0)=\Phi_{\ell, \alpha, Z^{\prime}}\left(0,0,\left(\Lambda^{\beta} \bar{u}(0,0,0)\right)_{|\beta| \leq k_{0}}\right) .
$$

Since $H$ is $k_{0}$-nondegenerate there are multi-indices $\alpha^{1}, \ldots, \alpha^{N^{\prime}}$ and $\ell^{1}, \ldots, \ell^{N^{\prime}} \in$ $\left\{1, \ldots, d^{\prime}\right\}$ such that if we set

$$
\Phi=\left(\Phi_{\ell^{1}, \alpha^{1}}, \ldots, \Phi_{\ell^{N^{\prime}}, \alpha^{N^{\prime}}}\right)
$$

then the matrix $\Phi_{Z^{\prime}}$ is invertible. Hence by Theorem 3.2 there is a smooth function $\phi=\left(\phi_{1}, \ldots, \phi_{N^{\prime}}\right)$ defined in a neighbourhood of $\left(0,\left(\Lambda^{\beta} \bar{u}(0,0,0)\right)_{|\beta|}\right)$ in $\mathbb{C}^{N^{\prime}} \times \mathbb{C}^{K_{0}}$ such that, if we shrink $U \times V$ accordingly,

$u_{j}(z, \bar{z}, s)=\phi_{j}\left(u(z, \bar{z}, s), \bar{u}(z, \bar{z}, s),\left(\Lambda^{\beta} \bar{u}(z, \bar{z}, s)\right)_{|\beta| \leq k_{0}}\right) \quad(z, s) \in U \times V, \quad j=1, \ldots, N^{\prime}$

and (3.3) holds. If we further shrink $U \times V$ and $\delta$ and choose $\Gamma^{\prime} \subset \subset \Gamma$ appropriately we see that

$$
g_{j}(z, \bar{z}, s, t)=\phi_{j}\left(h(z, \bar{z}, s,-t), \bar{h}(z, \bar{z}, s,-t),\left(\tilde{h}_{\ell, \beta}(z, \bar{z}, s, t)_{\ell \in\left\{1, \ldots, N^{\prime}\right\} ;|\beta| \leq k_{0}}\right)\right.
$$

is well defined for $t \in-\Gamma_{\delta}^{\prime}$. Here $\tilde{h}_{j, \beta}$ is the $\mathcal{M}$-almost-analytic extension of $\Lambda^{\beta} \bar{u}_{j}$ on $U \times V \times\left(-\Gamma_{\delta}^{\prime}\right)$, which exists due to (5.2), (3.6), (3.5) and [14, Theorem 4.4]. It is also easy to see that $\bar{h}(z, \bar{z}, s,-t)$ is $\mathcal{M}$-almost analytic on $U \times V \times\left(-\Gamma_{\delta}^{\prime}\right)$. We have that 


$$
\frac{\partial g_{j}}{\partial \bar{w}_{\ell}^{\prime}}=\sum_{k=1}^{N^{\prime}} \frac{\partial \phi_{j}}{\partial Z_{k}^{\prime}} \frac{\partial h_{k}}{\partial w_{\ell}^{\prime}}+\sum_{k=1}^{N^{\prime}} \frac{\partial \phi_{j}}{\partial \bar{Z}^{\prime}} \frac{\partial \bar{h}}{\partial w_{\ell}^{\prime}}+\sum_{k=1}^{N^{\prime}} \sum_{|\beta| \leq k_{0}} \frac{\partial \phi_{j}}{\partial W_{k, \beta}} \frac{\partial \tilde{h}_{k, \beta}}{\partial w_{\ell}^{\prime}}
$$

for $j=1, \ldots, N^{\prime}$ and $\ell=1, \ldots, d$. Note that we can choose $U \times V$ and $\Gamma_{\delta}^{\prime}$ so small that all functions appearing on the right-hand side are uniformly bounded. Hence, since $\partial_{w_{\ell}^{\prime}} \bar{h}=\overline{\partial_{\bar{w}_{\ell}^{\prime}}}$, the last two terms on the right-hand side of (5.5) are $\mathcal{M}$-almost analytic. The estimate (3.3) and the arguments in [25, Section 3.3] give that the first sum on the right-hand side of (5.5) is also $\mathcal{M}$-almost analytic. We conclude that $g_{j}$ is an $\mathcal{M}$-almost analytic extension on $U \times V \times\left(-\Gamma_{\delta}^{\prime}\right)$ of $u_{j}$ and thus

$$
\mathrm{WF}_{\mathcal{M}} u_{j} \subseteq(U \times V) \times\left(\mathbb{R}^{n} \times\left(\Gamma^{\prime} \cup-\Gamma^{\prime}\right)^{\circ}\right) \backslash\{0\}=(U \times V) \times\left(\mathbb{R}^{n} \backslash\{0\} \times\{0\}\right)
$$

On the other hand, since each $u_{j}$ is CR we have that $\left.\mathrm{WF}_{\mathcal{M}} u_{j}\right|_{0} \subseteq\{0\} \times \mathbb{R}^{d} \backslash\{0\}$ by (3.9) and we deduce that in fact $\left.\mathrm{WF}_{\mathcal{M}} u_{j}\right|_{0}=\emptyset$ for all $j=1, \ldots, N^{\prime}$. Hence the mapping $H$ is ultradifferentiable of class $\{\mathcal{M}\}$ near $p_{0}$.

In the rest of this section, it is always assumed that the weight sequence $\mathcal{M}$ is normal.

If we recall the well-known theorem of Tumanov [37] which states that any CR function on a minimal CR submanifold $M$ extends to a holomorphic function on a wedge with edge $M$, then we obtain the following corollary.

Corollary 5.3 Let $M \subseteq \mathbb{C}^{N}$ and $M^{\prime} \subseteq \mathbb{C}^{N^{\prime}}$ be generic submanifolds of class $\{\mathcal{M}\}$, $p_{0} \in M, p_{0}^{\prime} \in M^{\prime}, M$ minimal at $p_{0}$ and $H:\left(M, p_{0}\right) \rightarrow\left(M^{\prime}, p_{0}^{\prime}\right) a \mathcal{C}^{k_{0}}-C R$ mapping that is $k_{0}$-nondegenerate at $p_{0}$. Then $H$ is ultradifferentiable of class $\{\mathcal{M}\}$ in some neighbourhood of $p_{0}$.

This leads to the following result.

Corollary 5.4 Let $M \subseteq \mathbb{C}^{N}$ and $M^{\prime} \subseteq \mathbb{C}^{N^{\prime}}$ be generic submanifolds of class $\{\mathcal{M}\}$ that are both $k_{0}$-nondegenerate at $p_{0} \in M$ and $p_{0}^{\prime} \in M^{\prime}$, respectively. Furthermore assume that $M$ is minimal at $p_{0}$ and let $H: M \rightarrow M^{\prime}$ a CR diffeomorphism that is $\mathcal{C}^{k_{0}}$ near $p_{0}$ and satisfies $H\left(p_{0}\right)=p_{0}^{\prime}$. Then $H$ has to be ultradifferentiable of class $\{\mathcal{M}\}$ near $p_{0}$.

Recently, Berhanu-Xiao [3] showed that it is possible to slightly weaken the prerequisites of the smooth regularity result of Lamel. In particular, the source manifold $M$ can be chosen to be an abstract CR manifold. Using the methods developed previously we can also generalise this result to the ultradifferentiable category.

Theorem 5.5 Let $(M, \mathcal{V})$ be an abstract $C R$ manifold and $M^{\prime} \subseteq \mathbb{C}^{N^{\prime}}$ be a generic submanifold, both of class $\{\mathcal{M}\}$. Furthermore let $p_{0} \in M, H: M \rightarrow M^{\prime} a \mathcal{C}^{k_{0}}-C R$ mapping that is $k_{0}$-nondegenerate at $p_{0}$ and there is a closed acute cone $\Gamma \subseteq \mathbb{R}^{d}$ such that $\left.\mathrm{WF}_{\mathcal{M}} H\right|_{p_{0}} \subseteq\{0\} \times \Gamma$. Then $H$ is ultradifferentiable of class $\{\mathcal{M}\}$ near $p_{0}$.

Proof Since the assertion is local we will work on a small chart neighbourhood $\Omega=$ $U \times V \times W \subseteq \mathbb{R}^{n} \times \mathbb{R}^{n} \times \mathbb{R}^{d}$ of $M$ of $p_{0}=0$. Here $n$ denotes the CR dimension 
of $M$, whereas $d$ is the CR codimension of $M$. We use coordinates $(x, y, s)$ on $\Omega$ and write $z=x+i y$. In these coordinates a local basis of the CR vector fields of $M$ is given by

$$
L_{j}=\frac{\partial}{\partial \bar{z}_{j}}+\sum_{k=1}^{n} a_{j k} \frac{\partial}{\partial z_{k}}+\sum_{\alpha=1}^{d} b_{j \alpha} \frac{\partial}{\partial s_{\alpha}} \quad j=1, \ldots, n
$$

From the assumptions we conclude that if $\Omega$ is small enough that there is an open convex cone $\Gamma_{1} \subseteq \mathbb{R}^{N} \backslash\{0\}$ such that

$$
\mathrm{WF}_{\mathcal{M}} H=\bigcup_{j=1}^{N^{\prime}} \mathrm{WF}_{\mathcal{M}} H_{j} \subseteq \Omega \times \Gamma_{1}^{\circ}
$$

due to the closedness of $\mathrm{WF}_{\mathcal{M}} H$ in $T^{*} M \backslash\{0\}$. If we further shrink $\Omega$ (resp. $U, V$ and $W)$ and choose an open convex cone $\Gamma_{2} \subseteq \mathbb{R}^{N} \backslash\{0\}$ such that $\bar{\Gamma}_{2} \subseteq \Gamma_{1} \cup\{0\}$ we have by [14, Theorem 4.4] that there is an $\mathcal{M}$-almost extension $\tilde{F}$ with slow growth of $H$ onto $\Omega \times \Gamma_{2}$. If we now choose an open convex cone $\Gamma_{3} \subseteq \mathbb{R}^{d} \backslash\{0\}$ with $\{0\} \times \Gamma_{3} \subseteq \Gamma_{2}$, we infer that

$$
F:=\left.\tilde{F}\right|_{\Omega \times\left(\{0\} \times \Gamma_{3}\right)}
$$

is an $\mathcal{M}$-almost-analytic function on $U \times V \times W \times \Gamma_{3}$ with values in $\mathbb{C}^{N^{\prime}}$ and

$$
\lim _{\Gamma_{3} \ni t \rightarrow 0} F(., ., ., t)=H
$$

in the sense of distributions.

Let $\rho^{\prime}=\left(\rho_{1}^{\prime}, \ldots, \rho_{N^{\prime}}^{\prime}\right)$ be an ultradifferentiable defining function of $M^{\prime}$ near $p_{0}^{\prime}=H\left(p_{0}\right)$. As before in the proof of Theorem 1.1 we conclude that there are ultradifferentiable functions $\Phi_{\ell, \alpha}\left(Z^{\prime}, \bar{Z}^{\prime}, W\right)$ for $|\alpha| \leq k_{0}, \ell=1, \ldots, d^{\prime}$, defined in a neighbourhood of $\{0\} \times \mathbb{C}^{K_{0}} \subset \mathbb{C}^{N^{\prime}} \times \mathbb{C}^{K_{0}}$ and polynomial in the last $K_{0}=N^{\prime} \mid\{\alpha \in$ $\left.\mathbb{N}_{0}^{n^{\prime}}|| \alpha \mid \leq k_{0}\right\} \mid$ variables. From now on we can follow the proof of Theorem 1.1 verbatim.

\section{Ultradifferentiable Regularity of Infinitesimal CR Automorphisms}

In this section, we show how the results in [15] concerning the smoothness of infinitesimal CR automorphisms transfer to the ultradifferentiable setting. Since our presentation here differs in some details from that given in [15], we first recall the framework we are going to work in. In this section, $(M, \mathcal{V})$ is always an ultradifferentiable abstract $\mathrm{CR}$ manifold of class $\{\mathcal{M}\}$ with $\mathcal{M}$ being a normal weight sequence.

Definition 6.1 Let $U \subseteq M$ an open subset and $X: U \rightarrow T M$ a vector field of class $\mathcal{C}^{1}$. We say that $X$ is an infinitesimal CR automorphism iff its flow $H^{\tau}$, defined for 
small $\tau$, has the property, that there is $\varepsilon>0$ such that $H^{\tau}$ is a CR mapping provided that $|\tau| \leq \varepsilon$.

We need for the proofs of the regularity results a more suitable characterisation of infinitesimal CR automorphisms. We call a section $\mathfrak{Y} \in \Gamma\left(M,\left(T^{\prime} M\right)^{*}\right)$ a holomorphic vector field on $M$.

Apparently every vector field $X \in \Gamma(M, T M)$ gives rise to a holomorphic vector field by first extending $X$ to $\mathbb{C} T M$ and then restricting the extension to $T^{*} M$. For a partial converse, we recall from [15] the following purely algebraic result.

Lemma 6.2 Let $\mathfrak{Y} \in \Gamma\left(M,\left(T^{\prime} M\right)^{*}\right)$. Then there exists a unique vector field $X \in$ $\Gamma(M, T M)$ such that $\mathfrak{Y}$ is induced by $X$ if and only if $\mathfrak{Y}(\tau)=\overline{\mathfrak{Y}(\tau)}$ for all characteristic forms $\tau$.

From now on, we shall not distinguish between $X$ being a real vector field and a holomorphic vector field.

We recall the well-known identity, see e.g. [16],

$$
\mathcal{L}_{X} \alpha(Y)=d \alpha(X, Y)+Y \alpha(X)=X \alpha(Y)-\alpha([X, Y]),
$$

which holds for arbitrary complex vector fields $X, Y$ and complex forms $\alpha$ on smooth manifolds. We conclude that accordingly the Lie derivative

$$
\mathcal{L}_{L} \omega(.)=d \omega(L, .)
$$

of a holomorphic form $\omega$ with respect to a CR vector field $L$ is again a holomorphic form. It is now possible to make the following definition. We shall say that a holomorphic vector field $\mathfrak{Y} \in \Gamma\left(M,\left(T^{\prime} M\right)^{*}\right)$ is $\mathrm{CR}$ iff

$$
L \omega(\mathfrak{Y})=d \omega(L, \mathfrak{Y})
$$

for every CR vector field $L$ and holomorphic form $\omega$. In particular, a real vector field $X$ is CR if and only if

$$
\omega([L, X])=0
$$

for all CR vector fields $L$ and holomorphic forms $\omega$. We recall from [15] the following fact.

Proposition 6.3 If $X$ is an infinitesimal $C R$ automorphism on $M$, then $X$ considered as a holomorphic vector field, i.e. $X \in \mathcal{C}^{1}\left(M,\left(T^{\prime} M\right)^{*}\right)$ is $C R$.

We are now able to generalise the notion of infinitesimal CR automorphism. To this end consider the space $\mathcal{D}^{\prime}\left(M,\left(T^{\prime} M\right)^{*}\right)$ of distributions with values in $\left(T^{\prime} M\right)^{*}$.

Definition 6.4 An infinitesimal CR diffeomorphism of $M$ with distributional coefficients is a generalised holomorphic vector field $\mathfrak{Y} \in \mathcal{D}^{\prime}\left(M,\left(T^{\prime} M\right)^{*}\right)$ that satisfies

$$
L \omega(\mathfrak{Y})=\left(\mathcal{L}_{L} \omega\right)(\mathfrak{Y})
$$


for every CR vector field $L$ and holomorphic form $\omega$ and

$$
\mathfrak{Y}(\tau)=\overline{\mathfrak{Y}(\tau)}
$$

for all characteristic forms $\tau$.

Note that (6.1) is in fact a CR equation for $\mathfrak{Y}$. If $U \subseteq M$ is an open subset of $M$ then we say that $\mathfrak{Y} \in \mathcal{D}^{\prime}\left(M,\left(T^{\prime} M\right)^{*}\right)$ is an infinitesimal $\mathrm{CR}$ automorphism on $U$ iff (6.1) and (6.2) hold for all local sections $L \in \mathcal{E}_{\mathcal{M}}\left(U,\left.\mathcal{V}\right|_{U}\right)$ and $\theta \in \mathcal{E}_{\mathcal{M}}\left(U,\left.T^{0} M\right|_{U}\right)$, respectively. Let the subset $U \subseteq M$ be small enough such that there is a local basis $L_{1}, \ldots, L_{n}$ of CR vector fields and also a local basis $\omega^{1}, \ldots, \omega^{N}$ of the space of holomorphic forms. We recall that locally a distribution $\mathfrak{Y} \in \mathcal{D}^{\prime}\left(M,\left(T^{\prime} M\right)^{*}\right)$ is of the form

$$
\left.\mathfrak{Y}\right|_{U}=\sum_{j=1}^{N} X_{j} \omega^{j}
$$

with $X_{j} \in \mathcal{D}^{\prime}(U)$. We introduce also the following operators on $U$

$$
\mathbf{L}_{j}=L_{j} \cdot \mathbf{I} \mathbf{d}_{N}=\left(\begin{array}{ccc}
L_{j} & & 0 \\
& \ddots & \\
0 & & L_{j}
\end{array}\right)
$$

and note that since $d \omega^{k}\left(L_{j},.\right)$ is again a holomorphic form we have

$$
d \omega^{k}\left(L_{j}, .\right)=\sum_{\ell=1}^{N} B_{k, \ell}^{j} \omega^{\ell}
$$

with $B_{j, \ell}^{k} \in \mathcal{E}_{\mathcal{M}}(U)$. We observe that $\mathfrak{Y}$ is $\mathrm{CR}$ on $U$ if and only if

$$
\left.L_{j} X_{k}=L_{j}\left(\omega^{k}(\mathfrak{Y})\right)=d \omega^{k}\left(L_{j}, \mathfrak{Y}\right)\right)=\sum_{\ell=1}^{N} B_{k, \ell}^{j} X_{\ell}
$$

for all $1 \leq j \leq n$ and $0 \leq k \leq N$. We set

$$
B_{j}=\left(\begin{array}{ccc}
B_{j, 1}^{1} & \ldots & B_{j, N}^{1} \\
\vdots & & \vdots \\
B_{j, 1}^{N} & \ldots & B_{j, N}^{N}
\end{array}\right)
$$

Furthermore, using its local representation (6.3), we can identify $\mathfrak{Y}$ with the vector $X=\left(X_{1}, \ldots, X_{N}\right)$. Hence (6.1) turns into

$$
\mathbf{L}_{j} X=B_{j} \cdot X
$$


or

$$
P_{j} X=0
$$

respectively, where

$$
P_{j}=\mathbf{L}_{j}-B_{j}
$$

In particular, we infer from above and Theorem 3.5 that

$$
\mathrm{WF}_{\mathcal{M}} \mathfrak{Y} \subseteq T^{0} M
$$

Definition 6.5 Let $(M, \mathcal{V})$ be an ultradifferentiable abstract CR manifold of class $\{\mathcal{M}\}$, and $\mathfrak{Y}$ an infinitesimal $C R$ diffeomorphism of $M$ with distributional coefficients.

We say that $\mathfrak{Y}$ extends microlocally to a wedge with edge $M$ iff there exists a set $\Gamma \subseteq T^{0} M$ such that for each $p \in M$, the fibre $\Gamma_{p} \subseteq T_{p}^{0} M \backslash\{0\}$ is a closed, convex cone, and

$$
\mathrm{WF}_{\mathcal{M}}(\omega(\mathfrak{Y})) \subseteq \Gamma
$$

for every holomorphic form $\omega \in \mathcal{E}_{\mathcal{M}}\left(M, T^{\prime} M\right)$.

Theorem 6.6 Let $(M, \mathcal{V})$ be an ultradifferentiable abstract CR structure of class $\{\mathcal{M}\}$, and $\mathfrak{Y}$ an infinitesimal $C R$ diffeomorphism of $M$ with distributional coefficients which extends microlocally to a wedge with edge $M$. Then, for any $\omega \in E$, the evaluation $\omega(\mathfrak{Y})$ is ultradifferentiable, and for any $\lambda \in \mathcal{S}$, the vector field $\lambda \mathfrak{Y}$ is also of class $\{\mathcal{M}\}$.

Proof Since the assertion is local we will work in a suitable small open set $U \subseteq$ $M$ such that there are local bases $L_{1}, \ldots, L_{n}$ of $\mathcal{E}_{\mathcal{M}}(U, \mathcal{V})$ and $\omega^{1}, \ldots, \omega^{N}$ of $\mathcal{E}_{\mathcal{M}}\left(U, T^{\prime} M\right)$, respectively. We recall that we can represent $\mathfrak{Y}$ on $U$ by (6.3) or by $X=\left(X_{1}, \ldots, X_{N}\right) \in \mathcal{D}^{\prime}\left(U, \mathbb{C}^{N}\right)$. By assumption we know that there is a closed convex cone $\Gamma \subseteq T^{0} M \backslash\{0\}$ such that $\mathrm{WF}_{\mathcal{M}} X_{j} \subseteq \Gamma$ for each $j=1, \ldots, N$. If we set $W^{+}=(\Gamma)^{c} \subseteq T^{0} M \backslash\{0\}$, then $\mathrm{WF}_{\mathcal{M}} X_{j} \cap W^{+}=\emptyset$ for all $j=1, \ldots, N$. We may refer to this fact by saying that $X_{j}$ extends above. On the other hand, if we analogously put $W^{-}=(-\Gamma)^{c} \subseteq T^{0} M \backslash\{0\}$ then $\mathrm{WF}_{\mathcal{M}} \bar{X}_{j} \cap W^{-}=\emptyset$ by (3.6); we say that $\bar{X}_{j}$ extends below.

Furthermore, let $\left\{\theta^{1}, \ldots, \theta^{d}\right\}$ be a generating set of $\mathcal{E}_{\mathcal{M}}\left(U, T^{0} M\right)$ and recall (4.2), i.e.

$$
\mathcal{L}^{\alpha} \theta^{v}=\sum_{\ell=1}^{N} A_{\ell}^{\alpha, v} \omega^{\ell}
$$

with $A_{\ell}^{\alpha, v} \in \mathcal{E}_{\mathcal{M}}(U)$ for $\alpha \in \mathbb{N}_{0}^{n}$ and $v=1, \ldots, d$. In particular, (6.2), i.e. $\theta(\mathfrak{Y})=$ $\overline{\theta(\mathfrak{Y})}$, turns into 


$$
\sum_{\ell=1}^{N} A_{\ell}^{0, v} X_{\ell}=\sum_{\ell=1}^{N} \bar{A}_{\ell}^{0, v} \bar{X}_{\ell}
$$

and applying $\mathcal{L}^{\alpha}$ to (6.2) yields

$$
\sum_{\ell=1}^{N} A_{\ell}^{\alpha, v} X_{\ell}=\sum_{\ell=1}^{N} \sum_{|\beta| \leq|\alpha|} C_{\ell}^{\beta, v} L^{\beta} \bar{X}_{\ell},
$$

where $C_{\ell}^{\beta, \nu} \in \mathcal{E}_{\mathcal{M}}(U)$. Note that in both equations above the left-hand side extends above, while the right-hand side extends below.

Now choose any $N$-tuple $\underline{\alpha}=\left(\alpha^{1}, \ldots, \alpha^{N}\right) \in \mathbb{N}_{0}^{N n}$ of multi-indices with $|\alpha| \leq k$ for all $j=1, \ldots, N$ and $r=\left(r_{1}, \ldots, r_{N}\right) \in\{1, \ldots, d\}^{N}$. Then we have

$$
\left(\begin{array}{ccc}
A_{1}^{\alpha^{1}, r_{1}} & \ldots & A_{N}^{\alpha^{1}, r_{1}} \\
\vdots & \ddots & \vdots \\
A_{1}^{\alpha^{N}, r_{N}} & \ldots & A_{N}^{\alpha^{N}, r_{N}}
\end{array}\right)\left(\begin{array}{c}
X_{1} \\
\vdots \\
X_{N}
\end{array}\right)=\left(\begin{array}{c}
\sum C_{\beta}^{\alpha^{1}, \ell} L^{\beta} \bar{X}_{\ell} \\
\vdots \\
\sum C_{\beta}^{\alpha^{N}, \ell} L^{\beta} \bar{X}_{\ell}
\end{array}\right)
$$

If we multiply the equation with the classic adjoint of the matrix,

$$
\left(\begin{array}{ccc}
A_{1}^{\alpha^{1}, r_{1}} & \ldots & A_{N}^{\alpha^{1}, r_{1}} \\
\vdots & \ddots & \vdots \\
A_{1}^{\alpha^{N}, r_{N}} & \ldots & A_{N}^{\alpha^{N}, r_{N}}
\end{array}\right)
$$

then we obtain

$$
D(\underline{\alpha}, r) X_{j}=\sum_{\substack{|\beta| \leq k \\ \ell=1, \ldots, N}} D_{\beta, j}^{\underline{\alpha}, r} L^{\beta} \bar{X}_{j}
$$

for each $j=1, \ldots, N$ where the $D_{\beta, j}^{\underline{\alpha}, r}$ are ultradifferentiable functions on $U$. It follows that the right-hand side of this equation extends below, whereas the left-hand side obviously extends above. Hence $\mathrm{WF}_{\mathcal{M}} D(\underline{\alpha}, r) X=\emptyset$. We conclude that $\lambda X \in$ $\mathcal{E}_{\mathcal{M}}(U)$ for any $\lambda \in \mathcal{S}^{k}(U)$ since $\mathcal{S}^{k}(U)$ is generated by the functions $D(\underline{\alpha}, r)$.

The next statement is an obvious corollary of Theorem 6.6.

Corollary 6.7 Let $(M, \mathcal{V})$ be finitely nondegenerate and $X$ an infinitesimal CR diffeomorphism of $M$ with distributional coefficients which extends microlocally to a wedge with edge $M$. Then $X$ is ultradifferentiable of class $\{\mathcal{M}\}$.

However, the condition that $M$ is actually finitely nondegenerate is far too restrictive. We shall say that $(M, \mathcal{V})$ is CR regular if for every $p \in M$ there exists a multiplier $\lambda \in \mathcal{S}$ of the form $\lambda(x)=x^{\alpha} \tilde{\lambda}(x)$ and $\tilde{\lambda}(p) \neq 0$ in some local coordinates $x$ near $p$. Thence we can apply Proposition 2.12 . 
Theorem 6.8 Let $(M, \mathcal{V})$ be an abstract $C R$ structure, $p \in M$, and assume that $M$ is $C R$ regular near $p$. Then any locally integrable infinitesimal CR diffeomorphism $X$ of $M$ which extends microlocally to a wedge with edge $M$ is of class $\{\mathcal{M}\}$ near $p$.

In general it might be difficult to determine if a certain CR manifold is CR regular. In the following, we want to present some instances of CR regular manifolds. But first we take a closer look at the Lie derivatives of characteristic forms.

Suppose that $M$ is a CR manifold and near a point $p_{0} \in M$ there are local coordinates $(x, y, s)$ of $M$ such that the vector fields

$$
L_{j}=\frac{\partial}{\partial \bar{z}_{j}}-\sum_{\tau=1}^{d} b_{\tau}^{j} \frac{\partial}{\partial s_{\tau}}, \quad j=1, \ldots, n, z_{j}=x_{j}+y_{j}
$$

where $b_{\tau}^{j} \in \mathcal{E}_{\mathcal{M}}$, are a local basis of CR vector fields near $p_{0}$. In this setting (which includes, for example, generic manifolds with the local coordinates from Proposition 4.1 , see $[2, \S 1.6])$, the characteristic bundle is spanned by the forms

$$
\theta^{\tau}=d s_{\tau}+\sum_{j=1}^{n} b_{\tau}^{j} d \bar{z}_{j}+\sum_{j=1}^{n} \bar{b}_{\tau}^{j} d z_{j}, \quad \tau=1, \ldots, d .
$$

Furthermore, the forms $\theta^{\tau}, \tau=1, \ldots, d$ and $\omega^{j}=d z_{j}, j=1, \ldots, n$, constitute a local basis of holomorphic forms on $M$ near $p_{0}$. We also define the functions

$$
\lambda_{\mu}^{j, k}:=L_{k} \bar{b}_{\mu}^{j}-\bar{L}_{j} b_{\mu}^{k}
$$

for $j, k=1, \ldots, n$ and $\mu=1, \ldots, d$.

Consider a general holomorphic form

$$
\eta=\sum_{\mu=1}^{d} \sigma_{\mu} \theta^{\mu}+\sum_{j=1}^{n} \rho_{j} \omega^{j}
$$

The Lie derivative of $\eta$ with respect to the CR vector field $L_{k}$ is

$$
\mathcal{L}_{k} \eta=d \eta\left(L_{k}, .\right)=\sum_{\mu=1}^{d}\left(L_{k} \sigma_{\mu}-\sum_{\nu=1}^{d} \sigma_{\nu}\left(b_{\nu}^{k}\right)_{s_{\mu}}\right) \theta^{\mu}+\sum_{j=1}^{n}\left(L_{k} \rho_{j}+\sum_{\mu=1}^{d} \sigma_{\mu} \lambda_{\mu}^{j, k}\right) \omega^{j}
$$

Let $\left(\alpha=\left(\alpha_{1}, \cdots, \alpha_{n}\right) \in \mathbb{N}_{0}^{n}\right)$ be a multi-index of length $|\alpha|=m$. We introduce the finite sequence $m_{j}:=\sum_{\ell \leq j} \alpha_{\ell}, j=1, \ldots, n$, and set $m_{0}:=0$ and associate to $\alpha$ the function $p_{\alpha}:\{0,1, \ldots, m\} \rightarrow\{0,1, \ldots, n\}$ which is defined by

$$
p_{\alpha}(\ell)=j \quad \text { if } \ell \in\left(m_{j-1}, m_{j}\right]
$$

for $\ell=1, \ldots, m$ and $p_{\alpha}(0)=0$. We also associate the following sequences of multi-indices to $\alpha$ 


$$
\begin{array}{ll}
\alpha(\ell):=\sum_{q \leq \ell} e_{p_{\alpha}(q)} & \ell=0,1, \ldots, m, \\
\hat{\alpha}(\ell) & :=\sum_{q>\ell} e_{p(q)},
\end{array}
$$

where $e_{j}$ is the $j$-th standard unit vector in $\mathbb{R}^{n}$.

With this notation and (6.6), we can now state what the Lie derivative of the characteristic form $\theta^{\mu}(\mu=1, \ldots, d)$ is:

$$
\mathcal{L}^{\alpha} \theta^{\mu}=\sum_{\tau=1}^{d} T_{\tau}^{\alpha, \mu} \theta^{\tau}+\sum_{j=1}^{n} A_{j}^{\alpha, \mu} \omega^{j} .
$$

The functions $T_{\tau}^{\alpha, \mu}$ and $A_{j}^{\alpha, \mu}$ are defined iteratively by

$$
\begin{aligned}
& T_{\tau}^{0, \mu}=\delta_{\mu \tau}, \\
& T_{\tau}^{\alpha, \mu}=L_{p_{\alpha}(1)} T_{\tau}^{\hat{\alpha}(1), \mu}-\sum_{\nu=1}^{d}\left(b_{\nu}^{p(1)}\right)_{s_{\tau}} T_{\nu}^{\hat{\alpha}(1), \mu}
\end{aligned}
$$

and

$$
A_{j}^{\alpha, \mu}=\sum_{k=1}^{m} \sum_{\nu=1}^{d} L^{\alpha(k-1)}\left(T_{v}^{\alpha-\alpha(k), \mu} \lambda_{v}^{j, p_{\alpha}(k)}\right) .
$$

We are now able to give the first example of a CR regular submanifold of $\mathbb{C}^{N}$.

Definition 6.9 We say that a real hypersurface $M \subseteq \mathbb{C}^{N}$ is weakly nondegenerate at $p_{0}$ iff there exist coordinates $(z, w) \in \mathbb{C}^{n} \times \mathbb{C}$ near $p_{0}$ and numbers $k, m \in \mathbb{N}$ such that $p_{0}=0$ in these coordinates and $M$ is given near $p_{0}$ by an equation of the form

$$
\operatorname{Im} w=(\operatorname{Re} w)^{m} \varphi(z, \bar{z}, \operatorname{Re} w)
$$

where

$$
\frac{\partial^{|\alpha|} \varphi}{\partial z^{\alpha}}(0,0,0)=\frac{\partial^{|\alpha|} \varphi}{\partial \bar{z}^{\alpha}}(0,0,0)=0, \quad|\alpha| \leq k,
$$

and

$$
\operatorname{span}_{\mathbb{C}}\left\{\varphi_{z \bar{z}^{\alpha}}(0,0,0):|\alpha| \leq k\right\}=\mathbb{C}^{n}
$$

If $k_{0}$ is the smallest $k$ for which the preceding condition holds, we say that $M$ is weakly $k_{0}$-nondegenerate at $p_{0}$. 
Proposition 6.10 Let $M \subseteq \mathbb{C}^{N}$ be an ultradifferentiable real hypersurface, $p_{0} \in M$, and assume that $M$ is weakly $k_{0}$-nondegenerate at $p_{0}$. Then $M$ is $C R$ regular near $p_{0}$. In particular, any locally integrable infinitesimal $C R$ diffeomorphism of $M$ which extends microlocally to a wedge with edge $M$ near $p_{0}$ is ultradifferentiable near $p_{0}$.

Proof In order to show that $M$ is CR regular, we are going to construct a multiplier $\lambda \in \mathcal{S}$ of the form

$$
\lambda(z, \bar{z}, s)=s^{\ell} \psi(z, \bar{z}, s)
$$

in suitable local coordinates and with $\psi \in \mathcal{E}_{\mathcal{M}}$ not vanishing at $s=0$ and $\ell \in \mathbb{N}$.

By assumption there are coordinates $(z, w) \in \mathbb{C}^{n} \times \mathbb{C}$ such that $p_{0}=0$ and $M$ is given locally by

$$
\operatorname{Im} w=(\operatorname{Re} w)^{m} \varphi(z, \bar{z}, \operatorname{Re} w)
$$

where $m \in \mathbb{N}$ and $\varphi$ is an ultradifferentiable real-valued function defined near 0 with the property that $\varphi_{z^{\alpha}}(0)=\varphi_{\bar{z}^{\alpha}}(0)=0$ for $|\alpha| \leq k_{0}$ and

$$
\operatorname{span}_{\mathbb{C}}\left\{\varphi_{z \bar{z}^{\alpha}}(0,0,0): 0<|\alpha| \leq k_{0}\right\}=\mathbb{C}^{n}
$$

In these coordinates, a local basis of the $\mathrm{CR}$ vector fields on $M$ is given by

$$
L_{j}=\frac{\partial}{\partial \bar{z}_{j}}-b^{j} \frac{\partial}{\partial s}, \quad 1 \leq j \leq n,
$$

with

$$
b^{j}=i \frac{s^{m} \varphi_{\bar{z}_{j}}}{1+i\left(s^{m} \varphi\right)_{s}},
$$

whereas the characteristic bundle is spanned near the origin by

$$
\theta=d s+\sum_{j=1}^{n} b^{j} d \bar{z}_{j}+\sum_{j=1}^{n} b^{j} d z_{j}
$$

and $\theta$ together with the forms $\omega^{j}=d z_{j}$ constitute a local basis of $T^{\prime} M$ near the origin.

We observe that for $1 \leq j, \ell \leq n$

$$
\begin{aligned}
\lambda_{\ell}^{j}: & =L_{j} \bar{b}^{\ell}-\bar{L}_{\ell} b^{j} \\
= & s^{m}\left(\frac{i \varphi_{\bar{z}_{j \ell}}\left(1+i\left(s^{m} \varphi\right)_{s}\right)+\varphi_{z_{\ell}}\left(s^{m} \varphi_{\bar{z}_{j}}\right)_{s}}{\left(1+i\left(s^{m} \varphi\right)_{s}\right)^{2}}+\frac{\varphi_{\bar{z}_{j}}\left(\left(s^{m} \varphi_{z_{\ell}}\right)_{s}\left(1+i\left(s^{m} \varphi\right)_{s}\right)-i s^{m} \varphi_{z_{\ell}}\left(s^{m} \varphi\right)_{s s}\right)}{\left(1+i\left(s^{m} \varphi\right)_{s}\right)^{3}}\right. \\
& \left.\quad+\frac{i \varphi_{\bar{z}_{j} z_{\ell}}\left(1+i\left(s^{m} \varphi\right)_{s}\right)+\varphi_{\bar{z}_{j}}\left(s^{m} \varphi_{z_{\ell}}\right)_{s}}{\left(1+i\left(s^{m} \varphi\right)_{s}\right)^{2}}-\frac{\varphi_{z_{\ell}}\left(\left(s^{m} \varphi_{\bar{z}_{j}}\right)_{s}\left(1+i\left(s^{m} \varphi\right)_{s}\right)-s^{m} \varphi_{\bar{z}_{j}}\left(s^{m} \varphi\right)_{s s}\right)}{\left(1+i\left(s^{m} \varphi\right)_{s}\right)^{3}}\right) \\
& =s^{m} \chi_{\ell}^{j}
\end{aligned}
$$


and $\chi_{\ell}^{j}(0)=2 i \varphi_{\bar{z}_{j} z_{\ell}}(0)$ by the assumptions on $\varphi$.

In this setting, (6.7) takes the form

$$
\mathcal{L}^{\alpha} \theta=T^{\alpha} \theta+\sum_{j=1}^{n} A_{j}^{\alpha} \omega^{j}
$$

and (6.8) implies that

$$
\begin{aligned}
& T^{\alpha}=L_{p(1)} T^{\hat{\alpha}(1)}-\left(b^{p(1)}\right)_{s} T^{\hat{\alpha}(1)}, \quad T^{0}=1, \\
& A_{j}^{\alpha}=\sum_{k=1}^{|\alpha|}=L^{\alpha(k-1)}\left(T^{\hat{\alpha}(k)} \lambda_{p(k)}^{j}\right) .
\end{aligned}
$$

If we use two simple facts for smooth functions $f, g$, namely $\left(s^{q} f\right)_{s}=s^{q-1} f+$ $s^{q} f_{s}$ for $q \in \mathbb{N}$, we see that $T^{\beta}=s^{m-1} G^{\beta}$ for $|\beta| \geq 1$. Hence, if $m \geq 2$ we have

$$
A_{\ell}^{\alpha}(z, \bar{z}, s)=s^{m} \frac{2 i \varphi_{\bar{z}^{\alpha} z \ell}(z, \bar{z}, s)}{1+\left(s^{m} \varphi(z, \bar{z}, s)\right)_{s}^{2}}+s^{2 m-1} R_{\ell}^{\alpha}(z, \bar{z}, s)=s^{m} B_{\ell}^{\alpha}(z, \bar{z}, s) .
$$

On the other hand, we obtain for $m=1$ the following representation

$$
\begin{aligned}
A_{\ell}^{\alpha}(z, \bar{z}, s)= & s \frac{2 i \varphi_{\bar{z}^{\alpha} z \ell}(z, \bar{z}, s)}{1+\left(\varphi(z, \bar{z}, s)+s \varphi_{s}(z, \bar{z}, s)\right)^{2}} \\
& +s S_{\ell}^{\alpha}(z, \bar{z}, s)+s^{2} R_{\ell}^{\alpha}(z, \bar{z}, s)=s B_{\ell}^{\alpha}(z, \bar{z}, s),
\end{aligned}
$$

where $S_{\ell}^{\alpha}$ is a sum of products of rational functions with respect to $\varphi$ and its derivatives. Each of these summands contains at least one factor of the form $\varphi_{\bar{z}^{\beta}}$ or $\varphi_{z^{\beta}}$ with $|\beta| \leq|\alpha| \leq k_{0}$ and therefore $S_{\ell}^{\alpha}(0)=0$.

By assumption, there have to be multi-indices $\alpha^{1}, \ldots, \alpha^{n} \neq 0$ of length shorter than $k_{0}$ such that

$$
\left\{\varphi_{z \bar{z}^{\alpha^{1}}}(0), \ldots, \varphi_{z \bar{z}^{\alpha^{n}}}(0)\right\}
$$

is a basis for $\mathbb{C}^{n}$. Now we choose $\underline{\alpha}=\left(0, \alpha^{1}, \ldots, \alpha^{n}\right)$ and calculate according to (4.3) the multiplier $D(\underline{\alpha})=D(\underline{\alpha}, 1)($ note that $d=1)$ :

$$
\begin{aligned}
D(\underline{\alpha}) & =\operatorname{det}\left(\begin{array}{cccc}
1 & 0 & \ldots & 0 \\
A_{\theta}^{\alpha^{1}} & A_{1}^{\alpha^{1}} & \ldots & A_{n}^{\alpha^{1}} \\
\vdots & \vdots & \ddots & \vdots \\
A_{\theta}^{\alpha^{n}} & A_{1}^{\alpha^{n}} & \ldots & A_{n}^{\alpha^{n}}
\end{array}\right)=s^{n \cdot m} \operatorname{det}\left(\begin{array}{cccc}
1 & 0 & \ldots & 0 \\
A_{\theta}^{\alpha^{1}} & B_{1}^{\alpha^{1}} & \ldots & B_{n}^{\alpha^{1}} \\
\vdots & \vdots & \ddots & \vdots \\
A_{\theta}^{\alpha^{n}} & B_{1}^{\alpha^{n}} & \ldots & B_{n}^{\alpha^{n}}
\end{array}\right) \\
& =s^{n \cdot m} Q(\underline{\alpha}),
\end{aligned}
$$


where

$$
Q(\underline{\alpha})=\operatorname{det}\left(\begin{array}{cccc}
1 & 0 & \ldots & 0 \\
A_{\theta}^{\alpha^{1}} & B_{1}^{\alpha^{1}} & \ldots & B_{n}^{\alpha^{1}} \\
\vdots & \vdots & \ddots & \vdots \\
A_{\theta}^{\alpha^{n}} & B_{1}^{\alpha^{n}} & \ldots & B_{n}^{\alpha^{n}}
\end{array}\right)=\operatorname{det}\left(\begin{array}{ccc}
B_{1}^{\alpha^{1}} & \ldots & B_{n}^{\alpha^{1}} \\
\vdots & \ddots & \vdots \\
B_{1}^{\alpha^{n}} & \ldots & B_{n}^{\alpha^{n}}
\end{array}\right)
$$

hence

$$
Q(\underline{\alpha})(0)=(2 i)^{n} \operatorname{det}\left(\begin{array}{c}
\varphi_{z \bar{z}^{\alpha^{1}}}(0) \\
\vdots \\
\varphi_{z \bar{z}^{\alpha^{n}}}(0)
\end{array}\right) \neq 0 .
$$

We conclude that $M$ is $\mathrm{CR}$ regular.

Obviously, a similar approach as in the hypersurface case above can be used to find manifolds of higher codimension that are CR regular.

Definition 6.11 We say that a $\mathrm{CR}$ manifold $M \subseteq \mathbb{C}^{N}$ of codimension $d$ is weakly nondegenerate at $p_{0} \in M$ (in the first codimension) iff there are local coordinates $(z, w) \in \mathbb{C}^{n+d}$ near $p_{0}$ such that $M$ is given by the equations

$$
\operatorname{Im} w_{\mu}=(\operatorname{Re} w)^{\gamma^{\mu}} \varphi_{\mu}(z, \bar{z}, \operatorname{Re} w), \quad \mu=1, \ldots, d,
$$

with $\gamma^{1}<\gamma^{v}, v=2, \ldots, d$ and $\left|\gamma^{1}\right| \geq 2$. Furthermore, the function $\varphi_{1}$ satisfies for some $k$

$$
\operatorname{span}_{\mathbb{C}}\left\{\left(\varphi_{1}\right)_{z \bar{z}^{\alpha}}(0,0,0):|\alpha| \leq k\right\}=\mathbb{C}^{n}
$$

If $k_{0}$ is the smallest integer $k$ for which the above condition holds, we say that $M$ is weakly $k_{0}$-nondegenerate at $p_{0}$.

Proposition 6.12 Let $M \subseteq \mathbb{C}^{N}$ be a generic ultradifferentiable CR submanifold of codimension $d, p_{0} \in M$, and assume that $M$ is weakly nondegenerate at $p_{0}$. Then any locally integrable infinitesimal $C R$ diffeomorphism of $M$ which extends microlocally to a wedge with edge $M$ near $p_{0}$ is ultradifferentiable near $p_{0}$.

Proof Similar as before, we have to construct a multiplier $\lambda \in \mathcal{S}$ of the form $\lambda(z, \bar{z}, s)=s^{\beta} \psi(z, \bar{z}, s)$, where $\psi \in \mathcal{E}_{\mathcal{M}}$ and $\psi(0) \neq 0$. By assumption, there are coordinates $(z, w) \in \mathbb{C}^{n+d}$ near $p_{0}=0$ such that $M$ is given by

$$
\operatorname{Im} w_{\mu}=(\operatorname{Re} w)^{\gamma^{\mu}} \varphi_{\mu}(z, \bar{z}, \operatorname{Re} w), \quad \mu=1, \ldots, d .
$$

In particular, note that $\gamma^{1}<\gamma^{\mu}$ for $\mu=2, \ldots, d$. 
We use

$$
L_{j}=\frac{\partial}{\partial \bar{z}_{j}}-\sum_{\mu=1}^{d} b_{\mu}^{j} \frac{\partial}{\partial s_{\mu}}
$$

as a local basis of the CR vector fields near the origin. The coefficients $b_{\mu}^{j}$ are of the form

$$
b_{\mu}^{j}=i\left(\operatorname{det}\left(\operatorname{Id}_{d}+i \Phi\right)\right)^{-1} \cdot \operatorname{det} B_{\mu}^{j}
$$

where $\Phi$ denotes the Jacobi matrix of the map $\left(s^{\gamma^{\mu}} \varphi_{\mu}\right)_{\mu}$ with respect to the variables $s=\left(s_{1} \ldots, s_{d}\right)$ and

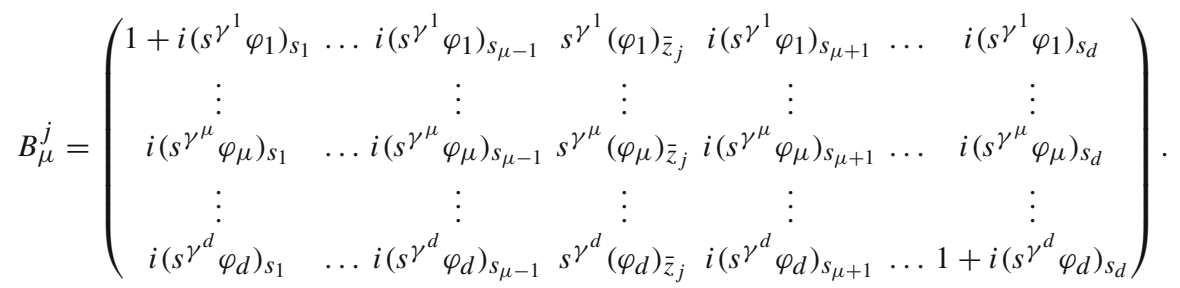

Hence for all $j=1, \ldots n$ and $\mu=1, \ldots, d$ we have

$$
b_{\mu}^{j}=i s^{\gamma^{1}}\left(\operatorname{det}\left(\operatorname{Id}_{d}+i \Phi\right)\right)^{-1} \operatorname{det} C_{\mu}^{j}
$$

with

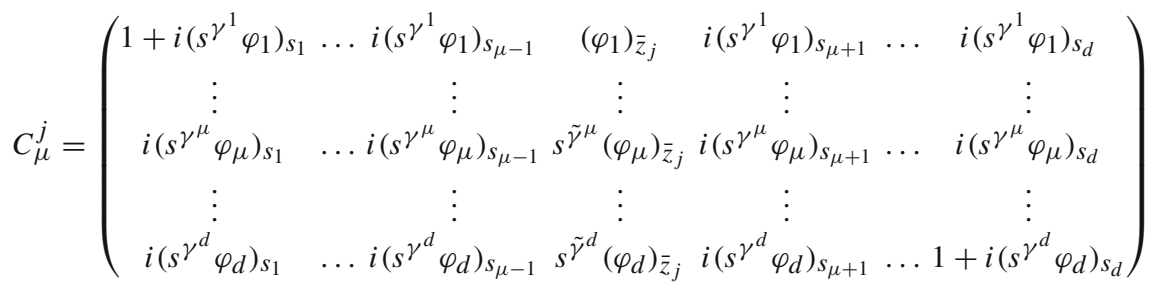

and $\tilde{\gamma}^{\mu}=\gamma^{\mu}-\gamma^{1}>0$. We observe that

$$
\left.\operatorname{det} C_{1}^{j}\right|_{s=0}=\left(\varphi_{1}\right)_{\bar{z}_{j}}(z, \bar{z}, 0) \quad \& \quad \operatorname{det} C_{\mu}^{j}=0, \quad \mu=2, \ldots, d \text {, }
$$

since $\left|\gamma^{\mu}\right| \geq\left|\gamma^{1}\right| \geq 2$. Furthermore, the forms

$$
\theta^{\mu}=d s_{\mu}+\sum_{j=1}^{n} b_{\mu}^{j} d \bar{z}_{j}+\sum_{j=1}^{n} \bar{b}_{\mu}^{j} d z_{j}, \quad \mu=1, \ldots, d,
$$


span the characteristic bundle near 0 and $\theta^{\mu}, \mu=1, \ldots, d$ and $\omega^{j}=d z_{j}, j=$ $1, \ldots, n$, form a local basis of the holomorphic forms on $M$. From (6.7), we recall for $\alpha \in \mathbb{N}_{0}^{n}$ and $\mu=1, \ldots, d$ that

$$
\mathcal{L}^{\alpha} \theta^{\mu}=\sum_{\tau=1}^{d} T_{\tau}^{\alpha, \mu} \theta^{\tau}+\sum_{j=1}^{n} A_{j}^{\alpha, \mu} \omega^{j}
$$

and from (6.8),

$$
\begin{aligned}
T_{\tau}^{0, \mu} & =\delta_{\mu \tau} \\
T_{\tau}^{\alpha, \mu} & =L_{p_{\alpha}(1)} T_{\tau}^{\hat{\alpha}(1), \mu}-\sum_{\nu=1}^{d}\left(b_{\nu}^{p(1)}\right)_{s_{\tau}} T_{v}^{\hat{\alpha}(1), \mu} \\
A_{j}^{\alpha, \mu} & =\sum_{k=1}^{|\alpha|} \sum_{\nu=1}^{d} L^{\alpha(k-1)}\left(T_{v}^{\alpha-\alpha(k), \mu} \lambda_{v}^{j, p_{\alpha}(k)}\right) .
\end{aligned}
$$

We recall that

$$
\lambda_{v}^{j, k}=L_{k} \bar{b}_{v}^{j}-\bar{L}_{j} b_{v}^{k}=\left(\bar{b}_{\nu}^{j}\right)_{\bar{z}_{k}}-\sum_{\mu=1}^{d} b_{\mu}^{k}\left(\bar{b}_{\nu}^{j}\right)_{s_{\mu}}-\left(b_{\nu}^{k}\right)_{z_{j}}+\sum_{\mu=1} \bar{b}_{\mu}^{j}\left(b_{\nu}^{k}\right)_{s_{\mu}}
$$

and note that (6.9) and (6.10) imply that

$$
\lambda_{v}^{j, k}=2 i s^{\gamma^{1}} R_{v}^{j, k} \quad v=1, \cdots, d,
$$

where

$$
\begin{array}{ll}
\left.R_{1}^{j, k}\right|_{s=0}=\left.\left(\varphi_{1}\right)_{\bar{z}_{k} z_{j}}\right|_{s=0} & \\
\left.R_{v}^{j, k}\right|_{s=0}=0 & v=1, \ldots, d .
\end{array}
$$

It is easy to see that also $\left.T_{\tau}^{\alpha, \mu}\right|_{s=0}=0$ for $\alpha \neq 0$. We conclude that for all $\alpha \neq 0$, and $j=1, \ldots, n$

$$
A_{j}^{\alpha, \mu}=2 i s^{\gamma^{1}} \tilde{A}_{j}^{\alpha, \mu} \quad \mu=1, \ldots, d
$$

where

$$
\begin{array}{ll}
\left.\tilde{A}_{j}^{\alpha, 1}\right|_{s=0}=\left.\left(\varphi_{1}\right)_{\bar{z}^{\alpha} z_{j}}\right|_{s=0}, & \\
\left.\tilde{A}_{j}^{\alpha, \mu}\right|_{s=0}=0 & \mu=2, \ldots, d .
\end{array}
$$


By assumption there are multi-indices $\alpha^{1}, \ldots, \alpha^{n} \in \mathbb{N}_{0}^{n}$ of length at most $k_{0}$ such that the vectors

$$
\left(\varphi_{1}\right)_{z \bar{z}^{\alpha j}}(0), \quad j=1, \ldots, n
$$

form a basis of $\mathbb{C}^{n}$.

We compute the multiplier $D(\bar{\alpha}, r)$ for $\underline{\alpha}=\left(0, \ldots, 0, \alpha^{1}, \ldots, \alpha^{n}\right)$ and $r=$ $(1,2, \ldots, d, 1, \ldots, 1)$. By (4.3) we have

$$
\begin{aligned}
& D(\underline{\alpha}, r)=\operatorname{det}\left(\begin{array}{cccccc}
1 & \ldots & 0 & 0 & \ldots & 0 \\
\vdots & & \vdots & \vdots & & \vdots \\
0 & \ldots & 1 & 0 & \ldots & 0 \\
T_{1}^{\alpha^{1}, 1} & \ldots & T_{d}^{\alpha^{1}, 1} & A_{1}^{\alpha^{1}, 1} & \ldots & A_{n}^{\alpha^{1}, 1} \\
\vdots & & \vdots & \vdots & & \vdots \\
T_{1}^{\alpha^{n}, 1} & \ldots & T_{d}^{\alpha^{n}, 1} & A_{1}^{\alpha^{n}, 1} & \ldots & A_{n}^{\alpha^{n}, 1}
\end{array}\right)
\end{aligned}
$$

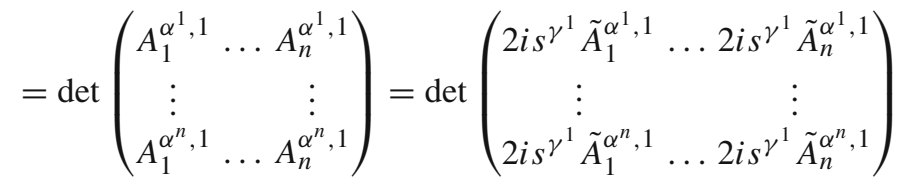

$$
\begin{aligned}
& =(2 i)^{n} s^{n \gamma^{1}} \operatorname{det}\left(\begin{array}{ccc}
\tilde{A}_{1}^{\alpha^{1}, 1} & \ldots & \tilde{A}_{n}^{\alpha^{1}, 1} \\
\vdots & & \vdots \\
\tilde{A}_{1}^{\alpha^{n}, 1} & \ldots & \tilde{A}_{n}^{\alpha^{n}, 1}
\end{array}\right)=(2 i)^{n} s^{n \gamma^{1}} \Lambda(\underline{\alpha}, r) \text {. }
\end{aligned}
$$

We conclude

$$
\Lambda(\underline{\alpha}, r)(0)=\operatorname{det}\left(\begin{array}{c}
\left(\varphi_{1}\right)_{z \bar{z}^{\alpha^{1}}}(0) \\
\vdots \\
\left(\varphi_{1}\right)_{z \bar{z}^{\alpha^{n}}}(0)
\end{array}\right) \neq 0
$$

In the preceding results, we required the involved manifolds to have a special form in order to simplify the necessary calculations, but of course there are many more $\mathrm{CR}$ regular manifolds. The next example gives a $\mathrm{CR}$ manifold that is not weakly nondegenerate at 0 in the sense of Definition 6.11 but is still CR regular.

Example 6.13 Let $M \subseteq \mathbb{C}^{3}$ be the $\mathrm{CR}$ manifold given by

$$
\begin{aligned}
& \operatorname{Im} w_{1}=\operatorname{Re} w_{1}|z|^{2}, \\
& \operatorname{Im} w_{2}=\operatorname{Re} w_{2}|z|^{2} .
\end{aligned}
$$


The CR bundle $\mathcal{V}$ of $M$ is spanned by

$$
L=\frac{\partial}{\partial \bar{z}}-i \frac{s_{1} z}{1+i|z|^{2}} \frac{\partial}{\partial s_{1}}-i \frac{s_{2} z}{1+i|z|^{2}} \frac{\partial}{\partial s_{2}} .
$$

Thus a basis of the characteristic form is given by

$$
\begin{aligned}
& \theta^{1}=d s_{1}+i \frac{s_{1} z}{1+i|z|^{2}} d \bar{z}-i \frac{s_{1} \bar{z}}{1-i|z|^{2}} d z \\
& \theta^{2}=d s_{2}+i \frac{s_{2} z}{1+i|z|^{2}} d \bar{z}-i \frac{s_{2} \bar{z}}{1-i|z|^{2}} d z
\end{aligned}
$$

We know that $\theta^{1}, \theta^{2}$ and $\omega=d z$ form a basis of $T^{\prime} M$. If $\alpha=e_{1}$ we recall from (6.7) that

$$
\mathcal{L}^{\alpha} \theta^{1}=T_{1}^{\alpha, 1} \theta^{1}+T_{2}^{\alpha, 1} \theta^{2}+A^{\alpha, 1} \omega
$$

Using (6.8), we observe that

$$
\begin{aligned}
T_{1}^{\alpha, 1} & =-i \frac{z}{1+i|z|^{2}} \\
T_{2}^{\alpha, 1} & =0 \\
A^{\alpha, 1} & =-2 i s_{1} \frac{1-|z|^{4}}{\left(1+|z|^{4}\right)^{2}} .
\end{aligned}
$$

Hence, if we set $\underline{\alpha}=(0,0, \alpha)$ and $r=(1,2,1)$ then the multiplier $D(\underline{\alpha}, r)$ of $M$ given by (4.3) is

$$
D(\underline{\alpha}, r)=\operatorname{det}\left(\begin{array}{ccc}
1 & 0 & 0 \\
0 & 1 & 0 \\
-i \frac{z}{1+i|z|^{2}} & 0 & -2 i s_{1} \frac{1-|z|^{4}}{\left(1+|z|^{4}\right)^{2}}
\end{array}\right)=-2 i s_{1} \frac{1-|z|^{4}}{\left(1+|z|^{4}\right)^{2}}
$$

and thus $M$ is $\mathrm{CR}$ regular.

We could now give an ultradifferentiable version of the example given in section 7 of [15] in order to show that in the previous statements the requirement on the infinitesimal automorphisms to be locally integrable is essential for the assertions to hold. However, to do this it would be enough to replace everywhere in section 7 of [15] the word smooth with the term ultradifferentiable of class $\{\mathcal{M}\}$.

Instead we take a closer look into the case of quasianalytic manifolds. We begin with recalling the following definition from [2, $\S 11.7]$. Let $M \subseteq \mathbb{C}^{N}$ be a CR submanifold with defining functions $\rho=\left(\rho_{1}, \ldots, \rho_{d}\right)$ near $p_{0} \in M$. A formal holomorphic vector field at $p_{0}$ is a vector field of the form 


$$
X=\sum_{j=1}^{N} a_{j}(Z) \frac{\partial}{\partial Z_{j}}
$$

with the coefficients $a_{j}$ being formal power series in $Z-p_{0}$ with complex coefficients. The formal vector field $X$ is said to be tangent to $M$ at $p_{0}$ iff there exists a $d \times d$ matrix $c(Z, \bar{Z})$ consisting of formal power series in the variables $Z-p_{0}$ and $\bar{Z}-\bar{p}_{0}$ such that

$$
X \rho(Z, \bar{Z}) \sim c(Z, \bar{Z}) \rho(Z, \bar{Z})
$$

where $\sim$ denotes equality as formal power series in $Z-p_{0}$ and $\bar{Z}-\bar{p}_{0}$. Note that the existence of nontrivial holomorphic vector fields at $p_{0}$ tangent to $M$ does not depend on the choice of holomorphic coordinates and defining equations near $p_{0}$.

Definition 6.14 A generic submanifold $M \subseteq \mathbb{C}^{N}$ is formally holomorphically nondegenerate at $p_{0} \in M$ iff there is no nontrivial formal holomorphic vector field at $p_{0}$ that is tangent to $M$.

Remark 6.15 If $M$ is formally holomorphically nondegenerate at $p_{0}$ then $M$ is formally holomorphically nondegenerate at every point of some neighbourhood $U$ of $p_{0}$. Furthermore, if $M$ is formally holomorphically nondegenerate on an open set $U \subseteq M$ then $M$ is finitely nondegenerate on an open and dense subset $V \subseteq U$, c.f. [2, Theorem 11.7.5].

Theorem 6.16 Let $\mathcal{M}$ be a quasianalytic normal weight sequence and $M \subseteq \mathbb{C}^{N} a$ generic submanifold of class $\{\mathcal{M}\}$ that is formally holomorphically nondegenerate. Every smooth $C R$ diffeomorphism $\mathfrak{Y}$ that extends microlocally to a wedge with edge $M$ is ultradifferentiable of class $\{\mathcal{M}\}$.

Proof As usual, we argue locally near a point $p_{0}$. After a choice of local bases of CR vector fields and holomorphic forms and selecting a generating set for the characteristic forms, we can use the representation (6.3) near $p_{0}$. By Theorem 6.6, we know that for any multiplier $\lambda$ the product $\Lambda_{j}=\lambda \cdot X_{j}$ is ultradifferentiable for $j=1, \ldots, N$. Since $X_{j}$ is smooth by assumption, we have that the equality holds also for the formal power series at $p_{0}$ of $\Lambda_{j}, \lambda$ and $X_{j}$. Since $M$ is formally holomorphically nondegenerate at $p_{0}$, there has to be a multiplier $\lambda \in \mathcal{S}$ with nontrivial formal power series at $p_{0}$. Indeed, if the power series of $\lambda$ at $p_{0}$ equals 0 then $\lambda$ itself has to vanish in a neighbourhood of $p_{0}$ by the quasianalyticity of $\mathcal{M}$. On the other hand, in every neighbourhood of $p_{0}$ there is a point $q$ at which $M$ is finitely nondegenerate by [2, Theorem 11.7.5]. Hence by Remark 4.5, there has to be a nontrivial multiplier $\lambda^{\prime}$ defined on some neighbourhood $U$ of $p_{0}$. We conclude that the formal power series of $\Lambda_{j}^{\prime}=\lambda^{\prime} X_{j}$ at $p_{0}$ is divisible by the Taylor series of $\lambda^{\prime}$ at $p_{0}$. Hence the main result in [29] gives that $X_{j}$ is ultradifferentiable of class $\{\mathcal{M}\}$ near $p_{0}$.

Acknowledgements Open access funding provided by Austrian Science Fund (FWF). The author was supported by the Austrian Science Fund FWF, international cooperation project Nr. I01776 and the Czech Science Foundation GACR Grant 17-19437S. The author would like to thank the anonymous referee for pointing out an error in the original version of Proposition 2.12. 
Open Access This article is distributed under the terms of the Creative Commons Attribution 4.0 International License (http://creativecommons.org/licenses/by/4.0/), which permits unrestricted use, distribution, and reproduction in any medium, provided you give appropriate credit to the original author(s) and the source, provide a link to the Creative Commons license, and indicate if changes were made.

\section{References}

1. Baouendi, M.S., Chang, C.H., Trèves, F.: Microlocal hypo-analyticity and extension of CR functions. J. Differ. Geom. 18(3), 331-391 (1983)

2. Baouendi, M.S., Ebenfelt, P., Rothschild, L.P.: Real submanifolds in complex space and their mappings. Princeton Mathematical Series, vol. 47. Princeton University Press, Princeton, NJ (1999)

3. Berhanu, S., Xiao, M.: On the $C^{\infty}$ version of the reflection principle for mappings between $\mathrm{CR}$ manifolds. Am. J. Math. 137(5), 1365-1400 (2015)

4. Bierstone, E., Milman, P.D.: Resolution of singularities in Denjoy-Carleman classes. Sel. Math. (N.S.) 10(1), 1-28 (2004)

5. Bony, J. M.: Équivalence des diverses notions de spectre singulier analytique. In: Séminaire GoulaouicSchwartz (1976/1977), Équations aux dérivées partielles et analyse fonctionnelle, Exp. No. 3, page 12. Centre Math., École Polytech., Palaiseau (1977)

6. Carleman, T.: Sur les fonctions indéfiniment dérivables. C. R. Acad. Sci. Paris 177, $422-424$ (1923)

7. Carleman, T.: Sur les fonctions quasi-analytiques. 5. Kongreß der Skandinav. Mathematiker in Helsingfors, 4.-7. Juli 1922. Helsingfors: Akadem. Buchh., pp. 181-196 (1923)

8. Chazarain, J., Piriou, A.: Introduction to the theory of linear partial differential equations, volume 14 of Studies in Mathematics and its Applications. North-Holland Publishing Co., Amsterdam-New York. Translated from the French (1982)

9. Denjoy, A.: Sur les fonctions quasi-analytiques de variable réelle. C. R. Acad. Sci. Paris 173, 1329-1331 (1921)

10. Dyn'kin, E. M.: Pseudoanalytic continuation of smooth functions. Uniform scale. Mathematical programming and related questions (Proc. Seventh Winter School, Drogobych, 1974), Theory of functions and functional analysis (Russian), pp. 40-73. Central Èkonom.-Mat. Inst. Akad. Nauk SSSR, Moscow (1976)

11. Dyn'kin, E.M.: Pseudoanalytic extension of smooth functions. The uniform scale. Am. Math. Soc. 115, 33-58 (1980)

12. Dyn'kin, E.M.: The pseudoanalytic extension. J. Anal. Math. 60, 45-70 (1993)

13. Fefferman, C.: The Bergman kernel and biholomorphic mappings of pseudoconvex domains. Invent. Math. 26, 1-65 (1974)

14. Fürdös, S.: Geometric microlocal analysis in Denjoy-Carleman classes. Submitted (2018)

15. Fürdös, S., Lamel, B.: Regularity of infinitesimal CR automorphisms. Internat. J. Math. 27(14), 1650112, 25pp (2016)

16. Helgason, S.: Differential geometry, Lie groups, and symmetric spaces, volume 34 of Graduate Studies in Mathematics. American Mathematical Society, Providence, RI, 2001. Corrected reprint of the 1978 original (2001)

17. Hörmander, L.: Uniqueness theorems and wave front sets for solutions of linear differential equations with analytic coefficients. Commun. Pure Appl. Math. 24, 671-704 (1971)

18. Hörmander, L.: A remark on Holmgren's uniqueness theorem. J. Differ. Geom. 6, 129-134, (1971/72)

19. Hörmander, L.: The analysis of linear partial differential operators. I. Classics in Mathematics. SpringerVerlag, Berlin (2003). Distribution theory and Fourier analysis, Reprint of the second (1990) edition

20. Huang, X.: Schwarz reflection principle in complex spaces of dimension two. Commun. Part. Differ. Equ. 21(11-12), 1781-1828 (1996)

21. Komatsu, H.: Ultradistributions. I. Structure theorems and a characterization. J. Fac. Sci. Univ. Tokyo Sect. IA Math. 20, 25-105 (1973)

22. Komatsu, H.: Ultradifferentiability of solutions of ordinary differential equations. Proc. Jpn. Acad. Ser. A 56(4), 137-142 (1980)

23. Lamel, B.: A reflection principle for real-analytic submanifolds of complex spaces. J. Geom. Anal. 11(4), 627-633 (2001) 
24. Lamel, B.: A $C^{\infty}$-regularity theorem for nondegenerate CR mappings. Monatsh. Math. 142(4), 315$326(2004)$

25. Lamel, B., Mir, N.: On the $\mathcal{C}^{\infty}$ regularity of CR mappings of positive codimension. Adv. Math. 335, 696-734 (2018)

26. Liess, O.: Carleman regularization in the $C^{\infty}$-category. Ann. Univ. Ferrara Sez. VII (N.S.) 45(suppl.), 213-240 (2000). 1999. Workshop on Partial Differential Equations (Ferrara, 1999)

27. Nagano, T.: Linear differential systems with singularities and an application to transitive Lie algebras. J. Math. Soc. Jpn. 18, 398-404 (1966)

28. Nirenberg, L., Webster, S., Yang, P.: Local boundary regularity of holomorphic mappings. Commun. Pure Appl. Math. 33(3), 305-338 (1980)

29. Nowak, K.J.: On division of quasianalytic function germs. Internat. J. Math. 24(13), 1350111, 5 (2013)

30. Pinčuk, S.I.: Holomorphic mappings of real-analytic hypersurfaces. Mat. Sbornik 105(147), 574-593, 640 (1978)

31. Rainer, A., Schindl, G.: Equivalence of stability properties for ultradifferentiable function classes. Rev. R. Acad. Cienc. Exactas Fís. Nat. 110(1), 17-32 (2016)

32. Rodino, L.: Linear Partial Differential Operators in Gevrey Spaces. World Scientific Publishing Co., Inc., River Edge, NJ (1993)

33. Sussmann, H.J.: Orbits of families of vector fields and integrability of distributions. Trans. Am. Math. Soc. 180, 171-188 (1973)

34. Thilliez, V.: Division by flat ultradifferentiable functions and sectorial extensions. Results Math. 44(12), 169-188 (2003)

35. Thilliez, V.: On quasianalytic local rings. Expo. Math. 26(1), 1-23 (2008)

36. Trèves, F.: Introduction to Pseudodifferential and Fourier Integral Operators. The University Series in Mathematics, vol. 2. Plenum Press, New York-London (1980)

37. Tumanov, A.E.: Extension of CR-functions into a wedge from a manifold of finite type. Mat. Sb. (N.S.) 136((178)(1)), 128-139 (1988)

38. Yamanaka, Takesi: On ODEs in the ultradifferentiable class. Nonlinear Anal. 17(7), 599-611 (1991)

Publisher's Note Springer Nature remains neutral with regard to jurisdictional claims in published maps and institutional affiliations. 